Research Article

\title{
Shigellosis Dynamics: Modelling the Effects of Treatment, Sanitation, and Education in the Presence of Carriers
}

\author{
Stephen Edward (D), ${ }^{1,2}$ Eunice Mureithi, ${ }^{1}$ and Nyimvua Shaban ${ }^{1}{ }^{1}$ \\ ${ }^{1}$ Department of Mathematics, University of Dar Es Salaam, Postal Address: Box 35062, Dar Es Salaam, Tanzania \\ ${ }^{2}$ Department of Mathematics and Statistics, University of Dodoma, Postal Address: Box 338, Dodoma, Tanzania \\ Correspondence should be addressed to Stephen Edward; stephenmwaihuti@yahoo.com
}

Received 15 January 2020; Revised 15 June 2020; Accepted 2 July 2020; Published 1 August 2020

Academic Editor: Jewgeni Dshalalow

Copyright (C) 2020 Stephen Edward et al. This is an open access article distributed under the Creative Commons Attribution License, which permits unrestricted use, distribution, and reproduction in any medium, provided the original work is properly cited.

\begin{abstract}
A mathematical model for Shigellosis including disease carriers with multiple control strategies is developed. We compute the effective reproductive number $R_{e}$, which is used to analyze the local stability of the equilibria, while the comparison theorem is used to prove global stability. By constructing a suitable Lyapunov function, the model endemic equilibrium is globally asymptotically stable when $R_{e}>1$. Sensitivity analysis is performed to investigate the parameters that have a high impact on the transmission dynamics of the disease with direct transmission contributing more infections than indirect transmission. The effects of control measures are then investigated both analytically and numerically. Numerical results show that there is a reduction in the number of infections when at least a single control measure is applied efficiently. However, as the number of control interventions increases, Shigellosis elimination is more possible. Results also show that carriers play a potential role in the prevalence of Shigellosis and ignoring these individuals could potentially undermine the efforts of containing this epidemic.
\end{abstract}

\section{Introduction}

Shigellosis is an enteric infectious disease which is caused by Shigella bacteria. These bacteria encompass four subgroups, namely, S. flexneri, S. sonnei, S. dysenteriae 1, and S. boydii [1]. It is responsible for approximately 1.1 million deaths per year worldwide. Approximately two-thirds of those who die from the disease are children under five years of age. It is one of the most common diarrhoea-related causes of morbidity and mortality in children in developing countries [2, 3]. Severe epidemics of dysentery can be caused by S. dysenteriae 1 which produces Shiga toxins, whereas the endemic form of the disease is caused essentially by $S$. flexneri and $S$. sonnei [4]. Shigellosis epidemics usually occur in areas with crowding and poor sanitary conditions, where direct transmission or contamination of food or water by the organism is common [5-12]. The disease is marked by fever, violent abdominal cramps, and rectal urgencies. Resistance to multiple antibiotics has recently been observed, including fluoroquinolones [13], which increases the threat of the occurrence of severe disease forms due to lack of efficient treatments. Unfortunately, no vaccine for the disease is available despite multiple and diverse vaccine design strategies $[14,15]$. Asymptomatic carriers pose a potential problem when it comes to controlling infectious diseases such as Shigellosis. The problem usually arises because carriers do not show clinical symptoms, as a result they continue infecting others unknowingly. Since they do not show symptoms, efforts to control the disease such as treatment and quarantine/isolation will ignore these individuals. On the contrary, initiatives such as vaccination will wrongly include these individuals because it is difficult to distinguish them from susceptible individuals. Therefore, it is necessary to explore the role played by the carrier in the transmission dynamics of Shigellosis infections.

Several scholars have studied Shigellosis in different ways including developing mathematical models (e.g., see [16-19]). Tien and Earn [16] developed a waterborne pathogen model termed as Susceptible-Infectious-Recovered-Water (SIRW); the model incorporated a dual 
transmission pathway with bilinear incidence rates employed for both the environment-to-human and humanto-human infection routes. They used the model to investigate the distinction between the different transmission routes in the dynamics of waterborne diseases. Chaturvedi et al. [17] studied Shigellosis by a SIRS model, with the assumption that transmission occurs solely via the personto-person pathway. Nonetheless, Shigellosis can also be contracted indirectly through person-to-environment or vice versa mainly, through food and water. Chen et al. [18] developed a Susceptible-Exposed-Asymptomatic-Infectious- Recovered-Water (SEARW) model that included a water compartment. Berhe et al. [19] developed an SIRB model that included a water compartment, but the model did not capture the role played by carriers and exposed individuals in Shigellosis transmission. Moreover, the model did not capture detection as an intervention in the study.

Most previous works have ignored the role played by carriers as such they could not capture interventions like screening (e.g., see [16-19]). Therefore, this study intends to explore the effects of control measures such as sanitation, treatment, and health education campaign on the dynamics of Shigellosis in the presence of carriers.

The rest of the paper is organized as follows. Section 2 focuses on model formulation, whereas Section 3 is based on the analysis of the model. In Section 4, the effects of control strategies are discussed. Numerical simulation is presented in Section 5, while Section 6 is devoted to sensitivity analysis, and lastly, Section 7 winds up by giving concluding remarks.

\section{Model Formulation}

The model considered here follows the basic SusceptibleExposed-Infected-Recovered (SEIR) model. This model is an extension of the work done by Tien and Earn [16]; in our case, we add extra classes for carriers and exposed. Indirect transmission is captured by nonlinear incidence function, contrary to previous models whose incidence were captured by linear functions. This is more realistic because nonlinear incidence function shows the presence of a gradual increase in disease incidence between the number of bacteria $(B)$ and the number of susceptible individuals $(S)$ than the counterpart which exhibits a sharp rise of incidence. On top of that, this ensures that the contact rate is bounded.

The total human population at time $t$ is subdivided into five mutually exclusive subpopulations: susceptible $(S(t))$, exposed $(E(t))$, infectious $(I(t))$, carrier $(C(t))$ (i.e., infected individuals who are contagious but do not show any disease symptoms), and recovered $(R(t))$. To incorporate a real biological phenomenon, an additional compartment, $B(t)$, which represents the reservoir of Shigella bacteria in the environment is considered.

It is assumed that susceptible individuals are recruited into the population at a constant rate, $\Lambda$. Susceptible individuals may acquire Shigella infection following effective direct contact with infectious individuals or carriers at the time-dependent rate $\lambda_{h}(t)$ or after ingesting environmental pathogens from contaminated aquatic reservoirs at the timedependent rate $\lambda_{p}(t)$. Here the term $\lambda_{h}(t)$ represents direct transmission between individuals, and it is modelled by standard mass action principle, whereas $\lambda_{p}(t)$ represents indirect transmission which is modelled by Holling type-II functional response (Michaelis-Menten function). These forces of infection are given by

$$
\begin{aligned}
& \lambda_{h}(t)=\beta_{1} I+\beta_{2} C, \\
& \lambda_{p}(t)=\frac{\phi B}{K+B},
\end{aligned}
$$

where $\beta_{1}=c_{1} q_{1}$ and $\beta_{2}=c_{2} q_{2}$ are the transmission rates for infectious and carrier individuals, respectively. We define $c_{1}, c_{2}$ as the number of contacts infectious and carrier individuals make with susceptibles per unit time, respectively, whereas $q_{1}, q_{2}$ are the probabilities that the contacts will cause infection. Likewise, $K$ is the half-saturation constant of the bacteria population in water that yields a $50 \%$ chance of catching the disease and $\phi>0$ is the ingestion rate of Shigella bacteria by individuals. The term $B /(K+B)$ represents the probability of a susceptible individual to develop Shigellosis per contact. It is assumed that the education campaign has an effect of reducing the number of Shigellosis infections. Therefore, both direct and indirect transmission will be reduced by the rate $(1-\rho)$, and thus the total force of infection will be given by $(1-\rho)\left(\lambda_{h}(t)+\lambda_{p}(t)\right)$, where $\rho \in[0,1]$ measures the efficacy of the education campaign. If $\rho=0$, then it implies that health education has been ignored as an intervention strategy, whereas when $\rho=1$, it means that education is $100 \%$ efficient in limiting the spread of Shigellosis. Exposed individuals may either join infectious or carrier classes. A fraction $q$ of the exposed individuals may progress to the infectious stage at the rate $\delta$ while the complement $(1-q)$ of the exposed may become carriers at the same rate $\delta$. Carrier individuals do not show any symptoms of Shigellosis even though they remain infectious; this complicates efforts to eliminate Shigellosis. A fraction $(1-l)$ of carriers are screened at the rate $\alpha$ and join the infectious individuals where they are finally treated at the rate $\gamma$. The remaining fraction $l$ of the carriers recover naturally at the rate $\eta_{2}$. Together with treatment measures, infectious individuals may experience natural recovery at the rate $\eta_{1}$. Shigellosis-induced mortality rate for infectious individuals is denoted by $d_{1}$, while the natural death rate of humans is represented by $\mu_{h}$. Shigellosis induces temporal immunity that wanes at the rate $\omega$. Therefore, recovered individuals may join the susceptible class when they lose their immunity. Infected individuals from both states $I(t)$ and $C(t)$ excrete bacteria into the environment at the reduced rates $(1-\rho) \epsilon_{1}$ and $(1-\rho) \epsilon_{2}$, respectively. It is assumed that the rate of excretion by the infectious individuals, $\epsilon_{1}$, is significantly higher than that by the carrier group, $\epsilon_{2}$. Note that despite low excretion of bacteria by the carrier group, because of its extremely long duration without showing any disease symptoms, the carrier group plays an important role in infection dynamics of Shigellosis. The per capita growth rate of Shigella bacteria is denoted by $r$ while bacteria deplete naturally at a rate $\mu_{b}$ or by sanitation measures at the rate $\sigma$. It is assumed that the growth rate of bacteria $(r)$ cannot exceed its death rate $\left(\mu_{b}\right)$ (that is, $\left.r<\mu_{b}\right)$. 
A full description of the variables and parameters to be used in the model is shown in Tables 1 and 2, respectively. The flow diagram for the dynamics is given in Figure 1.

From Figure 1, assumptions and model description the following system of differential equations are

$$
\begin{aligned}
& \frac{\mathrm{d} S}{\mathrm{~d} t}=\Lambda+\omega R-\left((1-\rho)\left(\lambda_{h}(t)+\lambda_{p}(t)\right)+\mu_{h}\right) S \\
& \frac{\mathrm{d} E}{\mathrm{~d} t}=(1-\rho)\left(\lambda_{h}(t)+\lambda_{p}(t)\right) S-\left(\mu_{h}+\delta\right) E \\
& \frac{\mathrm{d} I}{\mathrm{~d} t}=q \delta E+(1-l) \alpha C-\left(\mu_{h}+d_{1}+\eta_{1}+\gamma\right) I, \\
& \frac{\mathrm{d} C}{\mathrm{~d} t}=(1-q) \delta E-\left(\mu_{h}+l \eta_{2}+(1-l) \alpha\right) C, \\
& \frac{\mathrm{d} R}{\mathrm{~d} t}=\left(\eta_{1}+\gamma\right) I+l \eta_{2} C-\left(\mu_{h}+\omega\right) R, \\
& \frac{\mathrm{d} B}{\mathrm{~d} t}=r B\left(1-\frac{B}{K_{p}}\right)+(1-\rho) \epsilon_{1} I+(1-\rho) \epsilon_{2} C-\left(\mu_{b}+\sigma\right) B .
\end{aligned}
$$

The initial conditions for the model system (2) are $S(0)>0, E(0)>0, I(0)>0, C(0)>0, R(0)>0, B(0)>0$.

Model (2) is biologically meaningful in the invariant region

$$
\begin{aligned}
T= & \{S, E, I, C, R, B(t) \geq 0: S+E+I+C \\
& \left.+R \leq \frac{\Lambda}{\mu_{h}}, B(t) \leq \frac{(1-\rho)\left(\epsilon_{1}+\epsilon_{2}\right) \Lambda}{\mu_{h}\left(\mu_{b}+\sigma-r\right)}\right\} .
\end{aligned}
$$

\section{Analysis of the Model}

3.1. Existence of the Equilibrium Solutions. We establish the existence of the equilibrium points. To determine the equilibrium points, we set the right-hand side of system (2) to zero and solve the resulting system:

$$
\begin{aligned}
& 0=\Lambda+\omega R^{*}-\left((1-\rho) \lambda^{*}+\mu_{h}\right) S^{*} \\
& 0=(1-\rho) \lambda^{*} S^{*}-\left(\mu_{h}+\delta\right) E^{*} \\
& 0=q \delta E^{*}+(1-l) \alpha C-\left(\mu_{h}+d_{1}+\eta_{1}+\gamma\right) I^{*}, \\
& 0=(1-q) \delta E^{*}-\left(\mu_{h}+l \eta_{2}+(1-l) \alpha\right) C^{*}, \\
& 0=\left(\eta_{1}+\gamma\right) I^{*}+l \eta_{2} C^{*}-\left(\mu_{h}+\omega\right) R^{*}, \\
& 0=r B^{*}\left(1-\frac{B^{*}}{K_{p}}\right)+(1-\rho) \epsilon_{1} I^{*}+(1-\rho) \epsilon_{2} C^{*}-\left(\mu_{b}+\sigma\right) B^{*},
\end{aligned}
$$

where

$$
\lambda^{*}=\beta_{1} I^{*}+\beta_{2} C^{*}+\frac{\phi B^{*}}{K+B^{*}} .
$$

Solving $4^{\text {th }}$ equation of system (4), we get
TABLE 1: State variables and their description.

\begin{tabular}{lr}
\hline Variable & Description \\
\hline$S(t)$ & The number of susceptible humans at a time $t$. \\
$E(t)$ & The number of exposed humans at a time $t$. \\
$I(t)$ & The number of infectious humans at a time $t$. \\
$R(t)$ & The number of recovered (immune) humans at a time $t$. \\
$C(t)$ & The number of carriers at a time $t$. \\
$B(t)$ & The number of Shigella bacteria at a time $t$. \\
\hline
\end{tabular}

$$
C^{*}=\frac{(1-q) \delta E^{*}}{\mu_{h}+d_{2}+l \eta_{2}+(1-l) \alpha} .
$$

Substitute equation (6) into $3^{\text {rd }}$ equation of system (4) to get

$$
I^{*}=\frac{\left(q b_{2}+\alpha(1-l)(1-q)\right) \delta E^{*}}{\left(\mu_{h}+d_{1}+\eta_{1}+\gamma\right)\left(\mu_{h}+d_{2}+l \eta_{2}+(1-l) \alpha\right)} .
$$

Substitute equations (6) and (7) into fifth equation and solve for $R^{*}$ to get

$$
R^{*}=\frac{\left(\left(\eta_{1}+\gamma\right) Q_{2} \epsilon_{1}+l \eta_{2} Q_{1} \epsilon_{2}\right) E^{*}}{\mu_{h}+\omega}
$$

where

$$
\begin{aligned}
& Q_{1}=\frac{(1-q) \delta}{\mu_{h}+d_{2}+l \eta_{2}+(1-l) \alpha}, \\
& Q_{2}=\frac{\left(q b_{2}+\alpha(1-l)(1-q)\right) \delta}{\left(\mu_{h}+d_{1}+\eta_{1}+\gamma\right)\left(\mu_{h}+d_{2}+l \eta_{2}+(1-l) \alpha\right)} .
\end{aligned}
$$

From second equation of system (4), we have

$$
(1-\rho) \lambda^{*} S^{*}=\left(\mu_{h}+\delta\right) E^{*} .
$$

Substitute $R^{*}$ from equation (8) and $\lambda^{*} S^{*}$ from equation (10) into the first equation of system (4) to get

$$
S^{*}=\frac{\Lambda}{\mu_{h}}+\left(\frac{\left(\left(\eta_{1}+\gamma\right) Q_{2} \epsilon_{1}+\ln Q_{2} Q_{1} \epsilon_{2}\right) \omega}{\mu_{h}\left(\mu_{h}+\omega\right)}-\frac{\left(\mu_{h}+\delta\right)}{\mu_{h}}\right) E^{*} .
$$

Since the solution of system (4) is feasible only in the invariant region $T$, expression (11) can only be performed when $\quad\left(\left(\left(\eta_{1}+\gamma\right) Q_{2} \epsilon_{1}+l \eta_{2} Q_{1} \epsilon_{2}\right) \omega / \mu_{h}\left(\mu_{h}+\omega\right)\right)-\left(\left(\mu_{h}+\delta\right) /\right.$ $\left.\mu_{h}\right) \geq 0$, with equality at the disease-free equilibrium (DFE). From the $6^{\text {th }}$ equation of system (4), ignore the logistic growth of bacteria for simplicity to get

$$
\left(r-\left(\mu_{b}+\sigma\right)\right) B^{*}+\epsilon_{1} I^{*}+(1-\rho) \epsilon_{2} C^{*}=0 .
$$

Solving equation (12), we get

$$
B^{*}=g E^{*}, \text { where } g=\frac{(1-\rho) \epsilon_{1} Q_{2}+(1-\rho) \epsilon_{2} Q_{1}}{\mu_{b}+\sigma-r} .
$$

We know that the force of infection is given by

$$
\lambda^{*}=\beta_{1} I^{*}+\beta_{2} C^{*}+\frac{\phi B^{*}}{K+B^{*}} .
$$


Table 2: Parameters and their description.

\begin{tabular}{|c|c|c|c|}
\hline Parameter & Description & Value & Source \\
\hline$\Lambda$ & Rate of recruitment of individuals into the susceptible class & 462 humans/year & [19] \\
\hline$\beta_{1}$ & Transmission rate for infectious individuals & $0.4 /$ year & [19] \\
\hline$\beta_{2}$ & Transmission rates for carriers & $0.6 /$ year & [19] \\
\hline$\phi$ & Effective transmission rate of Shigellosis due to environment to human interaction & $0.4465 /$ year & [17] \\
\hline$\delta$ & Incubation rate (rate at which exposed individuals, $E(t)$, progress to either class $I(t)$ or $C(t)$ ) & $0.35 /$ day & {$[18]$} \\
\hline$\mu_{h}$ & Natural human mortality rate & $0.4465 /$ year & Assumed \\
\hline$q$ & A fraction of exposed individuals, $E(t)$, who progress to class $I(t)$ & 0.9 & {$[18]$} \\
\hline$l$ & A fraction of carriers, $C(t)$, who recover naturally and progress to class $R(t)$ & 0.4 & Assumed \\
\hline$\alpha$ & Rate of screening carriers & 0.56 & Assumed \\
\hline$d_{1}$ & Disease-induced death rate by $I(t)$ & $0.02 /$ year & Assumed \\
\hline$\eta_{1}$ & Recovery rate of infectious humans & $0.14 /$ day & [16] \\
\hline$\eta_{2}$ & Recovery rate of carrier humans & 0.0286/day & [18] \\
\hline$K_{p}$ & The environmental carrying capacity for Shigella bacteria in the food or water supply & $10^{4}$ cells $/ \mathrm{mL}$ & Assumed \\
\hline$K^{P}$ & Half-saturation rate of Shigella bacteria that can cause a $50 \%$ chance of infection & 60 cells $/ \mathrm{mL}$ & Assumed \\
\hline$\epsilon_{1}$ & Bacteria shed rate into the water supply by infectious human & 80 cells/mL/day & [20] \\
\hline$\epsilon_{2}$ & Bacteria shed rate into the water supply by carrier humans human & 70 cells/mL/day & [20] \\
\hline$r$ & Maximum per capita growth rate of Shigella bacteria & $0.73 /$ day & [21] \\
\hline$\mu_{b}$ & Mortality rate of Shigella bacteria, including phage degradation & $0.83 /$ day & Assumed \\
\hline$\omega$ & Waning rate of disease-induced immunity & $0.25 /$ year & [16] \\
\hline$\sigma$ & Rate at which sanitation leads to death of Shigella bacteria & $2 \mu_{b} /$ year & [22] \\
\hline$\gamma$ & Treatment rate for infectious individuals $(I)$ & $0.4 /$ year & Assumed \\
\hline$\rho$ & Education efficacy parameter & $0.6 /$ year & [21] \\
\hline
\end{tabular}

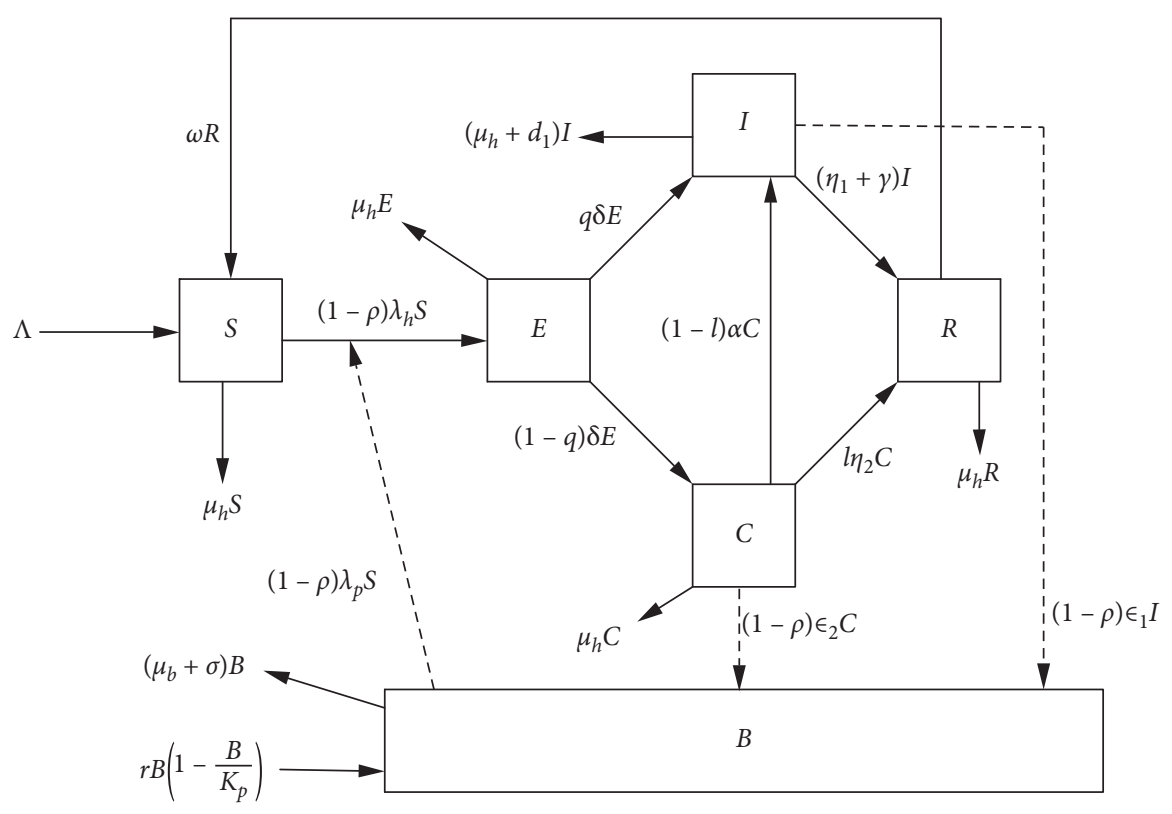

Figure 1: A flow diagram for Shigellosis transmission dynamics.

Substitute equations (6), (7), and (13) into equation (14) to get

$$
\lambda^{*}=\left(\beta_{1} Q_{2}+\beta_{2} Q_{1}+\frac{\phi g}{K+g E^{*}}\right) E^{*} .
$$

Substitute $\lambda^{*}$ from (15) and $S^{*}$ from (11) into second equation of system (4) to get

$$
(1-\rho)\left(\beta_{1} Q_{2}+\beta_{2} Q_{1}+\frac{\phi g}{K+g E^{*}}\right) E^{*}\left(\frac{\Lambda}{\mu_{h}}+\frac{h E^{*}}{\mu_{h}}\right)=\left(\mu_{h}+\delta\right) E^{*},
$$

resulting into a polynomial of degree three of the form of

$$
A_{2} E^{*^{3}}+A_{1} E^{*^{2}}+A_{0} E^{*}=0
$$


where

$$
\begin{aligned}
A_{0} & =\frac{\left(\mu_{h}+\delta\right) K \mu_{h}-\Lambda(1-\rho)\left(\beta_{1} K Q_{2}+\beta_{2} K Q_{1}+\phi \mathrm{g}\right)}{\mu_{h}}, \\
& =\left(\mu_{h}+\delta\right) K\left(1-R_{e}\right), \\
R_{e} & =\frac{(1-\rho) \Lambda\left(\beta_{1} K Q_{2}+\beta_{2} K Q_{1}+\phi \mathrm{g}\right)}{\mu_{h}\left(\mu_{h}+\delta\right) K}, \\
A_{1} & =\frac{\left(\mu_{h}+\delta\right) g \mu_{h}-(1-\rho)\left(\left(\beta_{1} Q_{2}+\beta_{2} Q_{1}\right)(g \Lambda+h K)+h \phi \mathrm{g}\right)}{\mu_{h}}, \\
& =\left(\mu_{h}+\delta\right) g\left(1-\frac{(1-\rho)\left(\left(\beta_{1} Q_{2}+\beta_{2} Q_{1}\right)(g \Lambda+h K)+h \phi \mathrm{g}\right)}{\mu_{h}\left(\mu_{h}+\delta\right) g}\right), \\
A_{2} & =\frac{g h(1-\rho)\left(\beta_{1} Q_{2}+\beta_{2} Q_{1}\right)}{\mu_{h}}, \\
h & =\frac{\left(\left(\eta_{1}+\gamma\right) Q_{2} \epsilon_{1}+l_{\eta_{2}} Q_{1} \epsilon_{2}\right) \omega}{\left(\mu_{h}+\omega\right)}-\left(\mu_{h}+\delta\right) .
\end{aligned}
$$

One of the solutions of equation (17) is $E^{*}=0$, which confirms the existence of disease-free equilibrium (DFE) while the existence of the endemic equilibrium (EE) is guaranteed by the nonzero solution $\left(E^{*}>0\right)$ of the quadratic equation:

$$
A_{2} E^{*^{2}}+A_{1} E^{*}+A_{0}=0 .
$$

To determine the DFE, substitute $E^{*}=0$ into equations (6)-(13) to get the DFE as

$$
E_{0}=\left(S^{0}, E^{0}, I^{0}, C^{0}, R^{0}, B^{0}\right)=\left(\frac{\Lambda}{\mu_{h}}, 0,0,0,0,0\right) .
$$

If $E^{*}>0$, then the EE denoted by $E_{1}$ is given by

$$
\begin{aligned}
E_{1} & =\left(S^{*}, E^{*}, I^{*}, C^{*}, R^{*}, B^{*}\right) \\
& =\left(\frac{\Lambda}{\mu_{h}}+\frac{h E^{*}}{\mu_{h}}, E^{*}, Q_{2} E^{*}, Q_{1} E^{*}, \frac{\left(\left(\eta_{1}+\gamma\right) Q_{2} \epsilon_{1}+l \eta_{2} Q_{1} \epsilon_{2}\right) E^{*}}{\mu_{h}+\omega}, g E^{*}\right),
\end{aligned}
$$

where $E^{*}$ is the positive solution of equation (19).

3.2. Reproduction Number. We compute the reproduction number, $R_{e}$, using the next-generation operator approach [23]. The reproduction number is obtained by taking the largest (dominant) eigenvalue (spectral radius) of the matrix

$$
F V^{-1}=\left[\frac{\partial \mathscr{F}_{i}\left(E_{0}\right)}{\partial x_{j}}\right]\left[\frac{\partial \mathscr{V}_{i}\left(E_{0}\right)}{\partial x_{j}}\right]^{-1}
$$

where $\mathscr{F}_{i}$ is the rate of appearance of new infection in compartment $i, \mathscr{V}_{i}$ is the transfer of infections from one compartment $i$ to another, and $E_{0}$ is the disease-free equilibrium. From system (2), we rewrite the equations with infectious classes, $E, I, C$, and $B$. This leads to the system

$$
\begin{aligned}
& \frac{\mathrm{d} E}{\mathrm{~d} t}=(1-\rho)\left(\lambda_{1}+\lambda_{2}\right) S-a_{0} E, \\
& \frac{\mathrm{d} I}{\mathrm{~d} t}=q \delta E+(1-l) \alpha C-b_{1} I,
\end{aligned}
$$$$
\frac{\mathrm{d} C}{\mathrm{~d} t}=(1-q) \delta E-b_{2} C,
$$$$
\frac{\mathrm{d} B}{\mathrm{~d} t}=r\left(1-\frac{B}{K_{b}}\right) B+(1-\rho) \epsilon_{1} I+(1-\rho) \epsilon_{2} C-\left(\mu_{b}+\sigma\right) B,
$$

where

$$
\begin{aligned}
& a_{0}=\mu_{h}+\delta, \\
& b_{1}=\mu_{h}+d_{1}+\eta_{1}+\gamma, \\
& b_{2}=\mu_{h}+l \eta_{2}+(1-l) \alpha .
\end{aligned}
$$

From system (23), we obtain

$$
\begin{aligned}
& \mathscr{F}_{i}=\left[\begin{array}{c}
(1-\rho)\left(\frac{\phi B S}{K+B}+\beta_{1} I S+\beta_{2} C S\right) \\
0 \\
0 \\
0
\end{array}\right], \\
& \mathscr{F}_{i}=\left[\begin{array}{c}
a_{0} E \\
b_{1} I-q \delta E-(1-l) \alpha C \\
b_{2} C-(1-q) \delta E \\
\left(\mu_{b}+\sigma\right) B-(1-\rho) \epsilon_{1} I-(1-\rho) \epsilon_{2} C-r\left(1-\frac{B}{K_{b}}\right) B
\end{array}\right] .
\end{aligned}
$$


Partial derivative of $\mathscr{F}_{i}$ and $\mathscr{V}_{i}$ with respect to $E, I, C$, and $B$ evaluated at $E_{0}$ gives

$$
F=\left[\begin{array}{cccc}
0 & (1-\rho) \beta_{1} S^{0} & (1-\rho) \beta_{2} S^{0} & (1-\rho) \frac{\phi S^{0}}{K} \\
0 & 0 & 0 & 0 \\
0 & 0 & 0 & 0 \\
0 & 0 & 0 & 0
\end{array}\right],
$$

$$
V=\left[\begin{array}{cccc}
a_{0} & 0 & 0 & 0 \\
-q \delta & b_{1} & -(1-l) \alpha & 0 \\
-(1-q) \delta & 0 & b_{2} & 0 \\
0 & -(1-\rho) \epsilon_{1} & -(1-\rho) \epsilon_{2} & -r+\mu_{b}+\sigma
\end{array}\right]
$$

The model reproduction number in the presence of control measures (treatment, education campaign, and sanitation) is now given by

$$
R_{e}=\rho\left(F V^{-1}\right)=R_{01}+R_{02}+R_{03}
$$

where

$$
\begin{aligned}
& R_{01}=\frac{\delta(1-\rho) S^{0}\left(b_{2} q+\alpha(1-l)(1-q)\right) \beta_{1}}{a_{0} b_{1} b_{2}}, \\
& R_{02}=\frac{\delta(1-\rho) S^{0}(1-q) \beta_{2}}{a_{0} b_{2}}, \\
& R_{03}=\delta(1-\rho) S^{0}\left(\frac{(1-\rho)\left(\epsilon_{1}\left(b_{2} q+\alpha(1-l)(1-q)\right)+b_{1}(1-q) \epsilon_{2}\right) \phi}{a_{0} b_{1} b_{2} K\left(\mu_{b}+\sigma-r\right)}\right) .
\end{aligned}
$$

Additionally, $R_{0 i}(i=1,2,3)$ are partial basic reproduction number induced by susceptible-to-infectious transmission, susceptible-to-carrier transmission, and environment-to-susceptible transmission, respectively.

3.2.1. The Basic Reproduction Number. In the absence of all the three control interventions, namely, treatment, education campaign, and sanitation, the basic reproduction is deduced from the reproduction number in equation (28) by setting $\rho=0, \sigma=0$, and $\gamma=0$. Therefore, the basic reproduction number is given by

$$
R_{0}=R_{1}^{\prime}+R_{2}^{\prime}+R_{3}^{\prime} \text {, }
$$

where

$$
\begin{aligned}
& R_{1}^{\prime}=\frac{\delta S^{0}\left(b_{2} q+\alpha(1-l)(1-q)\right) \beta_{1}}{a_{0}\left(\mu_{h}+d_{1}+\eta_{1}\right) b_{2}}, \\
& R_{2}^{\prime}=\frac{\delta S^{0}(1-q) \beta_{2}}{a_{0} b_{2}}, \\
& R_{3}^{\prime}=\delta S^{0}\left(\frac{\left(\epsilon_{1}\left(b_{2} q+\alpha(1-l)(1-q)\right)+\left(\mu_{h}+d_{1}+\eta_{1}\right)(1-q) \epsilon_{2}\right) \phi}{a_{0}\left(\mu_{h}+d_{1}+\eta_{1}\right) b_{2} K\left(\mu_{b}-r\right)}\right) .
\end{aligned}
$$

Each term $R_{i}^{\prime}(i=1,2,3)$ characterizes the contribution from infectious individuals, carriers, and environment, respectively, whereas $a_{0}$ and $b_{2}$ have been defined in equation (24).

\subsection{Stability Analysis of the Model Equilibria}

\subsubsection{Local Stability of the Disease-Free Equilibrium.}

Here we establish the stability of the DFE that is obtained in equation (20). This is stated in Theorem 1 as follows.

Theorem 1. The DFE of model (4) is locally asymptotically stable if $R_{e}<1$ and unstable if $R_{e}>1$.

Proof. The partial differentiation of system (4) with respect to $(S, E, I, C, R, B)$ at the DFE gives the Jacobian matrix $J$ as 


$$
J\left(E_{0}\right)=\left[\begin{array}{cccccc}
-\mu_{h} & 0 & -\beta_{1}(1-\rho) S^{0} & -\beta_{2}(1-\rho) S^{0} & -(1-\rho) \frac{\phi S^{0}}{K} & \omega \\
0 & -a_{0} & \beta_{1}(1-\rho) S^{0} & \beta_{2}(1-\rho) S^{0} & (1-\rho) \frac{\phi S^{0}}{K} & 0 \\
0 & q \delta & -b_{1} & (1-l) \alpha & 0 & 0 \\
0 & (1-q) \delta & 0 & -b_{2} & 0 & 0 \\
0 & 0 & (1-\rho) \varepsilon_{1} & (1-\rho) \varepsilon_{2} & r-\mu_{b}-\sigma & 0 \\
0 & 0 & \eta_{1}+\gamma & l \eta_{2} & 0 & -\left(\mu_{h}+\sigma\right)
\end{array}\right] \text {, }
$$

where $a_{0}, b_{1}$, and $b_{2}$ have been defined in equation (24).

Matrix (32) has two trivial negative eigenvalues $\lambda=-\mu_{h}$ and $\lambda=-\left(\mu_{h}+\sigma\right)$.

If we set $H_{11}=a_{0}, H_{12}=\beta_{1}(1-\rho) S_{0}, H_{13}=\beta_{2}(1-\rho) S_{0}$, $H_{14}=(1-\rho) S_{0} \phi / K, H_{21}=q \delta, \quad H_{22}=b_{1}, H_{23}=(1-l) \alpha$, $H_{31}=(1-q) \delta, H_{33}=b_{2}, H_{42}=(1-\rho) \epsilon_{1}, H_{43}=(1-\rho) \epsilon_{2}$, $H_{44}=\mu_{b}-r+\sigma$, then the remaining $4 \times 4$ submatrix is given as

$$
J_{1}\left(E_{0}\right)=\left[\begin{array}{cccc}
-H_{11} & H_{12} & H_{13} & H_{14} \\
H_{21} & -H_{22} & H_{23} & 0 \\
H_{31} & 0 & -H_{33} & 0 \\
0 & H_{42} & H_{43} & -H_{44}
\end{array}\right] .
$$

The remaining eigenvalues are the roots of the polynomial $\left|J_{1}\left(E_{0}\right)-\lambda\right|=0$, which is given by

$$
\lambda^{4}+c_{3} \lambda^{3}+c_{2} \lambda^{2}+c_{1} \lambda+c_{0}=0
$$

where the constants are such that

$$
\begin{aligned}
c_{3}= & H_{11}+H_{22}+H_{33}+H_{44}, \\
c_{2}= & H_{22} H_{33}+H_{11} H_{44}+H_{22} H_{44}+H_{33} H_{44} \\
& +H_{11} H_{22}\left(1-R_{g}\right)+H_{11} H_{33}\left(1-R_{d}\right), \\
c_{1}= & H_{11} H_{22} H_{44}\left(1-\left(R_{b}+R_{g}\right)\right)+H_{11} H_{33} H_{44}\left(1-\left(R_{c}+R_{d}\right)\right) \\
& +H_{11} H_{22} H_{33}\left(1-\left(R_{d}+R_{f}+R_{g}\right)\right)+H_{22} H_{33} H_{44}, \\
c_{0}= & H_{11} H_{22} H_{33} H_{44}\left(1-R_{e}\right) .
\end{aligned}
$$

Equivalently, $R_{e}$ can be split into parts

$$
R_{e}=R_{a}+R_{b}+R_{c}+R_{d}+R_{f}+R_{g} \text {, }
$$

where

$$
\begin{aligned}
& R_{a}=\frac{H_{14} H_{23} H_{31} H_{42}}{H_{11} H_{22} H_{33} H_{44}}, \\
& R_{b}=\frac{H_{14} H_{21} H_{42} H_{42}}{H_{11} H_{22} H_{44}}, \\
& R_{c}=\frac{H_{14} H_{31} H_{43} H_{42}}{H_{11} H_{33} H_{44}}, \\
& R_{d}=\frac{H_{13} H_{31}}{H_{11} H_{33}}, \\
& R_{f}=\frac{H_{12} H_{23} H_{31}}{H_{11} H_{22} H_{33} H_{44}}, \\
& R_{g}=\frac{H_{12} H_{21}}{H_{11} H_{22}} .
\end{aligned}
$$

To ensure that all roots of equation (34) have negative real parts, the Routh-Hurwitz stability criterion requires that

$$
\begin{aligned}
& c_{3}>0, \\
& c_{2}>0, \\
& c_{1}>0, \\
& c_{0}>0,
\end{aligned}
$$

$$
\begin{aligned}
D_{1} & =c_{3}>0, \\
D_{2} & =\left|\begin{array}{ll}
c_{3} & 1 \\
c_{1} & c_{2}
\end{array}\right|=c_{3} c_{2}-c_{1}>0, \\
D_{3} & =\left|\begin{array}{lll}
c_{3} & 1 & 0 \\
c_{1} & c_{2} & c_{3} \\
0 & c_{0} & c_{1}
\end{array}\right|=c_{1} c_{2} c_{3}-c_{1}^{2}-c_{0} c_{3}^{2}>0, \\
D_{4} & =\left|\begin{array}{llll}
c_{3} & 1 & 0 & 0 \\
c_{1} & c_{2} & c_{3} & 1 \\
0 & c_{0} & c_{1} & c_{2} \\
0 & 0 & 0 & c_{0}
\end{array}\right|=c_{0}\left(c_{1} c_{2} c_{3}-c_{1}^{2}-c_{0} c_{3}^{2}\right)>0 .
\end{aligned}
$$


It is obvious that $D_{1}=c_{3}>0$. In addition, if $R_{e}<1$, it implies that $R_{a}, R_{b}, R_{c}, R_{d}, R_{f}, R_{g}<1$, and hence $c_{0}, c_{1}, c_{2}>0$.

Also, $D_{2}$ can be shown to be positive as follows:

$$
\begin{aligned}
D_{2}= & c_{2} c_{3}-c_{1}=H_{11}\left(H_{22}\left(H_{44}\left(R_{b}+2\right)+H_{33}\left(R_{f}+2\right)\right)\right. \\
& \left.+H_{33} H_{44}\left(R_{c}+2\right)+H_{33}^{2}\left(1-R_{d}\right)+p_{1}\right) \\
& +H_{11}^{2}\left(H_{33}\left(1-R_{d}\right)+H_{22}\left(1-R_{g}\right)+H_{44}\right) \\
& +\left(H_{22}+H_{33}\right)\left(H_{22}+H_{44}\right)\left(H_{33}+H_{44}\right),
\end{aligned}
$$

where $p_{1}=H_{22}^{2}\left(1-R_{g}\right)+H_{44}^{2}$, and hence $D_{2}$ is positive.
The only remaining condition to show is

$$
D_{3}=c_{1}\left(c_{2} c_{3}-c_{1}\right)-c_{0} c_{3}^{2}>0 \text {. }
$$

To prove inequality (41), it is sufficient to establish the following two inequalities:

$$
\begin{gathered}
c_{1} c_{2} c_{3}>2 c_{1}^{2}, \\
c_{1} c_{2} c_{3}>2 c_{0} c_{3}^{2} .
\end{gathered}
$$

To show (42), we write $c_{2} c_{3}-2 c_{1}$ into the sum of the following parts:

$$
\begin{aligned}
c_{2} c_{3}-2 c_{1}= & 2 H_{22} H_{44} H_{11} R_{b}+2 H_{33} H_{44} H_{11} R_{c}+H_{33} H_{11}^{2}\left(1-R_{d}\right)+H_{33}^{2} H_{11}\left(1-R_{d}\right) \\
& +H_{22} H_{33} H_{11} R_{d}+H_{33} H_{44} H_{11} R_{d}+2 H_{22} H_{33} H_{11} R_{f}+H_{22} H_{11}^{2}\left(1-R_{g}\right) \\
& +H_{22}^{2} H_{11}\left(1-R_{g}\right)+H_{22} H_{33} H_{11} R_{g}+H_{22} H_{44} H_{11} R_{g}+H_{44} H_{11}^{2}+H_{22}^{2} H_{11} \\
& +H_{44}^{2} H_{11}+H_{22} H_{33} H_{11}+H_{22} H_{44} H_{11}+H_{33} H_{44} H_{11}+H_{22} H_{33}^{2}+H_{22} H_{44}^{2}+H_{33} H_{44}^{2} \\
& +H_{22}^{2} H_{33}+H_{22}^{2} H_{44}+H_{33}^{2} H_{44}+H_{22} H_{33} H_{44} .
\end{aligned}
$$

Similarly, to show (43), we write $c_{1} c_{2}-2 c_{0} c_{3}$ into the sum of parts as follows:

$$
\begin{aligned}
c_{1} c_{2}-2 c_{0} c_{3}= & H_{11} H_{22} H_{33}^{2} H_{44}\left(\left(1-R_{d}\right)+R_{c}+R_{f}+R_{g}+2 R_{a}+2 R_{b}\right) \\
& +H_{11} H_{22}^{2} H_{33} H_{44}\left(\left(1-R_{g}\right)+R_{b}+R_{d}+R_{f}+2 R_{a}+2 R_{c}\right) \\
& +H_{11}^{2} H_{22} H_{33} H_{44}\left(1-\left(R_{d}+R_{g}\right)+2 R_{a}+R_{b}+R_{c}+R_{f}+R_{b} R_{d}+2 R_{d} R_{g}+R_{c} R_{g}\right) \\
& +H_{11}^{2} H_{22}^{2} H_{33}\left(1-\left(R_{d}+R_{f}+R_{g}\right)+R_{d} R_{g}+R_{f} R_{g}+R_{g}^{2}\right) \\
& +H_{11} H_{22}^{2} H_{33}^{2}\left(1-R_{d}-R_{f}-R_{g}\right)+H_{11}^{2} H_{22} H_{33}^{2}\left(1-R_{d}\right)\left(1-R_{d}-R_{f}-R_{g}\right) \\
& +H_{11} H_{22}^{2} H_{44}^{2}\left(1-R_{b}-R_{g}\right)+H_{11}^{2} H_{22} H_{44}^{2}\left(1-R_{b}-R_{g}\right) \\
& +H_{11}^{2} H_{22}^{2} H_{44}\left(1-R_{g}\right)\left(1-R_{b}-R_{g}\right)+H_{11} H_{33}^{2} H_{44}^{2}\left(1-R_{c}-R_{d}\right) \\
& +H_{11}^{2} H_{33}^{2} H_{44}\left(1-R_{d}\right)\left(1-R_{c}-R_{d}\right)+H_{11}^{2} H_{33} H_{44}^{2}\left(1-R_{c}-R_{d}\right)+H_{22} H_{33}^{2} H_{44}^{2} \\
& +H_{22}^{2} H_{33}^{2} H_{44}+H_{11} H_{22} H_{33} H_{44}^{2}\left(1+R_{b}+R_{c}+R_{d}+R_{g}+2 R_{a}+2 R_{f}\right) \\
& +H_{22}^{2} H_{33} H_{44}^{2} .
\end{aligned}
$$

It can be noted that if $R_{e}<1$, then each $R_{a}, R_{b}, R_{c}, R_{d}, R_{f}, R_{g}<1$ and therefore $c_{1} c_{2}-2 c_{0} c_{3}>0$ and $c_{2} c_{3}-2 c_{1}>0$. Thus, equations (42) and (43) hold and so does condition (41). In the same fashion, the proof for condition $D_{4}$ can be established from the fact that $D_{4}=c_{0} D_{3}$. Fortunately, we have already proved that $D_{3}>0$; therefore, it is clear that $D_{4}=c_{0} D_{3}>0$. Hence, all conditions of Routh-Hurwitz for this case (equations (38) and (39)) are satisfied; then, the disease-free equilibrium $E_{0}$ is locally asymptotically stable whenever $R_{e}<1$.
3.3.2. Global Stability of the Disease-Free Equilibrium. We have the following results on the global stability of the DFE.

Theorem 2. If $R_{e}<1$, the DFE is globally asymptotically stable and unstable if $R_{e}>1$.

Proof. By the comparison theorem, the rate of change of the variables representing the infected components of the model system (2) can be rewritten as 


$$
\left(\begin{array}{c}
\frac{\mathrm{d} E}{\mathrm{~d} t} \\
\frac{\mathrm{dI}}{\mathrm{dt}} \\
\frac{\mathrm{d} C}{\mathrm{~d} t} \\
\frac{\mathrm{d} B}{\mathrm{~d} t}
\end{array}\right)=(F-V)\left(\begin{array}{c}
E \\
I \\
B
\end{array}\right)-\left(\begin{array}{cccc}
0 & (1-\rho) \beta_{1} S^{0} & (1-\rho) \beta_{2} S^{0} & (1-\rho) \frac{\phi S^{0}}{K+B} \\
0 & 0 & 0 & 0 \\
0 & 0 & 0 & 0 \\
0 & 0 & 0 & 0
\end{array}\right)\left(\begin{array}{c}
E \\
I \\
B \\
C \\
\end{array}\right),
$$

implying that

$$
\left(\begin{array}{c}
\frac{\mathrm{d} E}{\mathrm{~d} t} \\
\frac{\mathrm{d} I}{\mathrm{~d} t} \\
\frac{\mathrm{d} C}{\mathrm{~d} t} \\
\frac{\mathrm{d} B}{\mathrm{~d} t}
\end{array}\right) \leq(F-V)\left(\begin{array}{c}
E \\
I \\
C \\
B
\end{array}\right),
$$

where $F$ and $V$ are Jacobian matrices as in (26) and (27). Since the eigenvalues of the matrix $(F-V)$ have negative real parts (this comes from the stability results in Theorem $1)$, system (2) is stable whenever $R_{e}<1$. So, $(S, E, I, C, B, R) \longrightarrow\left(S^{0}, 0,0,0,0,0\right)$ and $S \longrightarrow S_{0}$ as $t \longrightarrow \infty$. By the comparison theorem (see [24]), $(S, E, I, C, B, R) \longrightarrow E_{0}$ as $t \longrightarrow \infty$. Therefore, $E_{0}$ is globally asymptotically stable whenever $R_{e}<1$.

\subsubsection{Global Stability of the Endemic Equilibrium}

Theorem 3. The endemic equilibrium for the model (2) is globally asymptotically stable on $T$ if $R_{e}>1$.

Proof. Here we construct an explicit Lyapunov function of the form

$$
L=\sum_{i=1}^{n} w_{i}\left(x_{i}-x_{i}^{*} \ln x\right)
$$

where $w_{i}$ is a properly selected positive constant, $x_{i}$ is the population of the $i^{\text {th }}$ compartment, and $x_{i}^{*}$ is the equilibrium level. We define the Lyapunov function candidate $L$ for model system (2) as

$$
\begin{aligned}
L= & w_{1}\left(S-S^{*} \ln S\right)+w_{2}\left(E-E^{*} \ln E\right)+w_{3}\left(I-I^{*} \ln I\right) \\
& +w_{4}\left(C-C^{*} \ln C\right)+w_{5}\left(R-R^{*} \ln R\right)+w_{6}\left(B-B^{*} \ln B\right) .
\end{aligned}
$$
by

The time derivative of the Lyapunov function $L$ is given

$$
\begin{aligned}
\frac{\mathrm{d} L}{\mathrm{~d} t}= & w_{1}\left(1-\frac{S^{*}}{S}\right) \frac{\mathrm{d} S}{\mathrm{~d} t}+w_{2}\left(1-\frac{E^{*}}{E}\right) \frac{\mathrm{d} E}{\mathrm{~d} t}+w_{3}\left(1-\frac{I^{*}}{I}\right) \frac{\mathrm{d} I}{\mathrm{~d} t} \\
& +w_{4}\left(1-\frac{C^{*}}{C}\right) \frac{\mathrm{d} C}{\mathrm{~d} t} \\
& +w_{5}\left(1-\frac{R^{*}}{R}\right) \frac{\mathrm{d} R}{\mathrm{~d} t}+w_{6}\left(1-\frac{B^{*}}{B}\right) \frac{\mathrm{d} B}{\mathrm{~d} t} .
\end{aligned}
$$

It can be noted that at endemic equilibrium (see equation (4)), we have

$$
\begin{aligned}
& \Lambda=-\omega R^{*}+\left((1-\rho) \lambda^{*}+\mu_{h}\right) S^{*}, \\
& a_{0}=\frac{(1-\rho) \lambda^{*} S^{*}}{E^{*}}, \\
& b_{1}=\frac{q \delta E^{*}+(1-l) \alpha C^{*}}{I^{*}}, \\
& b_{2}=\frac{(1-q) \delta E^{*}}{C^{*}}, \\
& b_{3}=\frac{\left(\eta_{1}+\gamma\right) I^{*}+l \eta_{2} C^{*}}{R^{*}}, \\
& b_{4}=\frac{r B^{*}\left(1-\left(B^{*} / K_{p}\right)\right)+(1-\rho) \epsilon_{1} I^{*}+(1-\rho) \epsilon_{2} C^{*}}{B^{*}},
\end{aligned}
$$

and substituting equation (51) into equation (50) and simplifying can result into the following equation: 


$$
\begin{aligned}
\frac{\mathrm{d} L}{\mathrm{~d} t}= & w_{1} \omega R\left(1-\frac{S^{*}}{S}\right)\left(1-\frac{R^{*}}{R}\right)-w_{1}(1-\rho) \lambda S\left(1-\frac{S^{*}}{S}\right)\left(1-\frac{\lambda^{*} S^{*}}{\lambda S}\right) \\
& -w_{1} \mu_{h} S\left(1-\frac{S^{*}}{S}\right)^{2}+w_{2}(1-\rho) \lambda S\left(1-\frac{E^{*}}{E}\right)\left(1-\frac{\lambda^{*} S^{*} E}{\lambda S E^{*}}\right) \\
& +w_{3} q \delta E\left(1-\frac{I^{*}}{I}\right)\left(1-\frac{E^{*} I}{E I^{*}}\right)+w_{3}(1-l) \alpha C\left(1-\frac{I^{*}}{I}\right)\left(1-\frac{C^{*} I}{C I^{*}}\right) \\
& +w_{4}(1-q) \delta E\left(1-\frac{C^{*}}{C}\right)\left(1-\frac{E^{*} C}{E C^{*}}\right)+w_{5}\left(\eta_{1}+\gamma\right) I\left(1-\frac{R^{*}}{R}\right)\left(1-\frac{I^{*} R}{I R^{*}}\right) \\
& +w_{5} l \eta_{2} C\left(1-\frac{R^{*}}{R}\right)\left(1-\frac{C^{*} R}{C R^{*}}\right)-\frac{w_{6} R B^{2}}{K_{p}}\left(1-\frac{B^{*}}{B}\right)^{2} \\
& +w_{6}(1-\rho) \epsilon_{1} I\left(1-\frac{B^{*}}{B}\right)\left(1-\frac{I^{*} B}{I B^{*}}\right)+w_{6}(1-\rho) \epsilon_{2} C\left(1-\frac{B^{*}}{B}\right)\left(1-\frac{C^{*} B}{C B^{*}}\right) \\
= & -w_{1} \mu_{h} S\left(1-\frac{S^{*}}{S}\right)^{2}-\frac{w_{6} R B^{2}}{K_{p}}\left(1-\frac{B^{*}}{B}\right)^{2}+F(S, E, I, C, R, B),
\end{aligned}
$$

where $F$ is the balance of the right-hand terms of equation (52). Following the approach by [25-27], $F$ is a nonpositive function for $S, E, I, C, R, B>0$. Thus, $\mathrm{d} L / \mathrm{d} t<0$ for $S, E, I, C, R, B>0$ and is zero if $S=S^{*}, E=E^{*}, I=I^{*}, C=$ $C^{*}, R=R^{*}$ and $B=B^{*}$. Therefore, if $R_{e}>1$, model (2) has a unique endemic equilibrium point $E_{1}$ which is globally asymptotically stable.

\section{Effects of Control Intervention Strategies}

We investigate the impacts of implementing control interventions, either singly or in a combination. Most governments have to invest in facilities for combating Shigellosis such as sanitation services, ensuring the availability of drugs in health care centres as well as emphasizing on education campaigns on how to avoid the disease. So, it is imperative to have a clear understanding of the benefits of implementing a different combination of intervention strategies. Different countries have different economic status as such a different ability to deal with diseases. Most low-income countries are unable to overcome various diseases such as Shigellosis because they invest fewer resources to overcome it. However, rich countries have eliminated or have minor cases of waterborne diseases like Shigellosis since they can target all possible means of transmission and are prepared in advance to tackle this epidemic once it erupts. Reproductive thresholds for all possible cases ranging from single through three control intervention were calculated and compared among themselves. The primary purpose of the comparison was to determine which of them has a significant influence in diminishing Shigellosis.

4.1. Effects of Multiple Control Intervention Strategies. We focus on the effects of a combination of two or three strategies simultaneously.

4.1.1. Effects of Treatment, Education Campaign, and Sanitation. In the presence of three interventions, that is, $\rho \neq 0, \sigma \neq 0$, and $\gamma \neq 0$, the reproduction number-induced by treatment, education, and sanitation

$$
R_{e}=R_{01}+R_{02}+R_{03}
$$

where $R_{01}, R_{02}$, and $R_{03}$ have been defined in equation (29).

4.1.2. Effects of Treatment and Education Campaign. In the absence of sanitation $(\sigma=0)$, the treatment and educationinduced reproduction number is

$$
R_{\mathrm{Ted}}=R_{01}+R_{02}+R_{03}^{\prime \prime} \text {, }
$$

where

$$
R_{03}^{\prime \prime}=\delta(1-\rho) S^{0}\left(\frac{(1-\rho)\left(\epsilon_{1}\left(b_{2} q+\alpha(1-l)(1-q)\right)+b_{1}(1-q) \epsilon_{2}\right) \phi}{a_{0} b_{1} b_{2} K\left(\mu_{b}-r\right)}\right)
$$

where $R_{01}$ and $R_{02}$ have been defined in equation (29) and $R_{03}^{\prime \prime}$ represents the contribution from the surroundings to
Shigellosis transmissions in absence of sanitation effort but in the presence of education and medical treatment. Together 
with that, $a_{0}, b_{1}$, and $b_{2}$ have been defined in equation (24). Since $\mu_{b}+\sigma-r>\mu_{b}-r$, then $R_{03}<R_{03}^{\prime \prime}$, and hence $R_{e}<R_{\mathrm{Ted}}$, which shows that even though treatment and education campaigns can reduce the spread of infections, multiple control strategy that accounts for all three controls $\left(R_{e}\right)$ will yield a better result.

4.1.3. Effects of Sanitation and Treatment. In the absence of education campaign $(\rho=0)$, the sanitation and treatmentinduced reproduction number is

$$
R_{\mathrm{ST}}=R_{1}+R_{2}+R_{3} \text {, }
$$

where

$$
\begin{aligned}
& R_{1}=\frac{\delta S^{0}\left(b_{2} q+\alpha(1-l)(1-q)\right) \beta_{1}}{a_{0} b_{1} b_{2}}, \\
& R_{2}=\frac{\delta S^{0}(1-q) \beta_{2}}{a_{0} b_{2}}, \\
& R_{3}=\delta S^{0}\left(\frac{\left(\epsilon_{1}\left(b_{2} q+\alpha(1-l)(1-q)\right)+b_{1}(1-q) \epsilon_{2}\right) \phi}{a_{0} b_{1} b_{2} K\left(\mu_{b}+\sigma-r\right)}\right),
\end{aligned}
$$

where $R_{1}, R_{2}$, and $R_{3}$ represent the contributions from infectious individuals, carriers, and environment-to-host transmission, respectively. It must be noted that $a_{0}, b_{1}$, and $b_{2}$ have been defined in equation (24). It is possible to express $\quad R_{01}=(1-\rho) R_{1}, R_{02}=(1-\rho) R_{2}, R_{03}=(1-\rho)^{2} R_{3}$. Since $\rho \in[0,1]$, then it is clear to see that $R_{01} \leq R_{1}, R_{02} \leq R_{2}$ and $R_{03} \leq R_{3}$; therefore, $R_{e}<R_{\mathrm{ST}}$. This shows that sanitation and treatment alone are not sufficient to eliminate Shigellosis infections; there is a need for an additional control intervention strategy to bring the disease to an end. The strategy that takes care of all three controls $\left(R_{e}\right)$ will yield a far better result. This result agrees with intuitive expectations.

4.1.4. Effects of Sanitation and Education. In the absence of treatment $(\gamma=0)$, the sanitation and education-induced reproduction number is

$R_{\mathrm{Sed}}=R_{01}^{\prime}+R_{02}+R_{03}^{\prime}$,

where

$$
\begin{aligned}
& R_{01}^{\prime}=\frac{\delta(1-\rho) S^{0}\left(b_{2} q+\alpha(1-l)(1-q)\right) \beta_{1}}{a_{0}\left(\mu_{h}+d_{1}+\eta_{1}\right) b_{2}} \\
& R_{03}^{\prime}=\frac{\delta(1-\rho)^{2} S^{0}\left(\epsilon_{1}\left(b_{2} q+\alpha(1-l)(1-q)\right)+\left(\mu_{h}+d_{1}+\eta_{1}\right)(1-q) \epsilon_{2}\right) \phi}{a_{0}\left(\mu_{h}+d_{1}+\eta_{1}\right) b_{2} K\left(\mu_{b}+\sigma-r\right)},
\end{aligned}
$$

where $R_{01}^{\prime}$ and $R_{03}^{\prime}$ represent the contributions from infectious individuals and contribution from environment-to-host transmission, respectively, whereas $R_{02}$ has been defined in equation (29). Since $\mu_{h}+d_{1}+\eta_{1}<b_{1}$, then $R_{01}^{\prime}>R_{01}$ and $R_{03}^{\prime}>R_{03}$; therefore, $R_{e}<R_{\text {Sed }}$. The result shows that once the strategy that combines sanitation and education campaigns is implemented thoroughly in the community, it can reduce the severity of the disease to a certain extent. However, when all three control interventions are implemented $\left(R_{e}\right)$, the result is more appealing.

4.2. Effects of Single Control Intervention Strategy. We focus on the effects of each of the controls individually.

4.2.1. Effects of Treatment. In the absence of education campaign $(\rho=0)$ and sanitation $(\sigma=0)$, the treatment-induced reproduction number is

$$
R_{T}=R_{1}+R_{2}+R_{3}^{\prime \prime}
$$

where

$$
R_{3}^{\prime \prime}=\delta S^{0}\left(\frac{\left(\epsilon_{1}\left(b_{2} q+\alpha(1-l)(1-q)\right)+b_{1}(1-q) \epsilon_{2}\right) \phi}{a_{0} b_{1} b_{2} K\left(\mu_{b}-r\right)}\right)
$$

where $R_{3}^{\prime \prime}$ represents the contribution from the surroundings to Shigellosis transmissions in absence of sanitation effort, whereas, $R_{1}, R_{2}$ have been defined in equation (57). From equation (59), it has been shown that $R_{1}>R_{01}, R_{2}>R_{02}$. Since $\left(1 /\left(\mu_{b}+\sigma-r\right)\right)<1 /\left(\mu_{b}-r\right)$, it implies that $R_{3}<R_{3}^{\prime \prime}$; hence, $R_{\mathrm{ST}}<R_{T}$. Since $R_{e}<R_{\mathrm{ST}}$, we can summarize the inequality as $R_{e}<R_{\mathrm{ST}}<R_{T}$. This inequality suggests that three control strategies are far better than two strategies, and two strategies are far better than single strategy.

4.2.2. Effects of Education Campaign. In the absence of treatment $(\gamma=0)$ and water purification $(\sigma=0)$, the education-induced reproduction number is

$$
R_{\mathrm{ed}}=R_{01}^{\prime}+R_{2}+R_{03}^{\prime \prime},
$$

where 


$$
R_{03}^{\prime \prime}=\delta(1-\rho) S^{0}\left(\frac{(1-\rho)\left(\epsilon_{1}\left(b_{2} q+\alpha(1-l)(1-q)\right)+\left(\mu_{h}+d_{1}+\eta_{1}\right)(1-q) \epsilon_{2}\right) \phi}{a_{0}\left(\mu_{h}+d_{1}+\eta_{1}\right) b_{2} K\left(\mu_{b}-r\right)}\right),
$$

where $R_{03}^{\prime \prime}$ represents the contribution from the surroundings to Shigellosis transmissions and $R_{01}^{\prime}$ is defined in equation (59), whereas $R_{2}$ is defined in equation (57).
4.2.3. Effects of Sanitation Strategy. In the absence of treatment $(\gamma=0)$ and education campaigns $(\rho=0)$, the sanitation-induced reproduction number is

$$
R_{S}=R_{1}^{\prime}+R_{2}+R_{3}^{\prime \prime}
$$

where

$$
R_{3}^{\prime \prime}=\delta S^{0}\left(\frac{\left(\epsilon_{1}\left(b_{2} q+\alpha(1-l)(1-q)\right)+\left(\mu_{h}+d_{1}+\eta_{1}\right)(1-q) \epsilon_{2}\right) \phi}{a_{0}\left(\mu_{h}+d_{1}+\eta_{1}\right) b_{2} K\left(\mu_{b}+\sigma-r\right)}\right)
$$

where $R_{3}^{\prime \prime}$ represents the contribution from the surroundings to Shigellosis transmissions, whereas $R_{1}^{\prime}$ is defined in (30) and $R_{2}$ is defined in equation (57). It can be seen that $R_{e}<R_{S}$ since $R_{1}^{\prime}>R_{01}, R_{2}>R_{02}$ and $R_{3}^{\prime \prime}>R_{03}$. Besides that, one can note that $R_{3}^{\prime}<R_{3}^{\prime}$, which implies $R_{S}<R_{0}$. We use numerical simulations (Figure 2) to compare all reproduction numbers.

\section{Numerical Simulations}

In this section, the model system (2) was solved numerically by using the Runge-Kutta order four schemes due to the fact that they provide more stable solutions as compared with Euler's method. Euler's method is inadequate even for well-conditioned problems if a high degree of accuracy is required, owing to the slow first-order convergence. So, it is generally more convenient to use Runge-Kutta fourthorder methods. The aim was to validate the analytical results obtained in the previous sections. The implementation of the scheme was done using MATLAB package. Plots of the numerical solution are used to investigate the effect of some parameters on the population component of interest. The parameters used for simulation are shown in Table 2, while initial values for subpopulations are given as follows: $\quad S(0)=9, E(0)=7, I(0)=8, C(0)=6, R(0)=6$, and $B(0)=7$.

From Figure 3, if the screening rate $\alpha=30 \%$, then the number of infectious individuals (I) decreases, but the number of asymptomatic carriers is still high. This is not a desirable result because asymptomatic carriers are responsible for most of the new infections since they are unaware of their illness. When $\alpha$ is increased from $30 \%$ to $60 \%$ and lastly to $90 \%$, the number of asymptomatic carriers shows a much more significant decline while the number of symptomatic infectious remains low. This shows that high testing coverage of Shigellosis could contribute to the detection of infectious individuals, thereby mitigating the transmission dynamics of the disease.

Figure 4 shows that an increase in $\phi$ or $\beta_{1}$ has an effect of increasing $R_{e}$ and hence the number of infections. One can note that the change in $\beta_{1}$ causes a sharp rise $R_{e}$ while the corresponding change in $\phi$ causes a minimal change $R_{e}$ which means that direct transmission plays a significant role in the transmission dynamics of Shigellosis as compared with the indirect transmission.

From Figure 2, one can see the comparison between the strategies, in particular, Figure 2(a) shows the comparison among single strategies from which treatment strategy $\left(R_{T}\right)$ is the best-case scenario followed by a strategy that takes care of sole education campaign $\left(R_{\mathrm{ed}}\right)$ and the worst case being sanitation strategy $\left(R_{S}\right)$. This implies that Shigellosis can be better controlled with treatment efforts than with education or sanitation. Though this does not mean that the education campaign and sanitation are useless, their effectiveness is less than treatment. It must be acknowledged that Shigellosis suffers drug resistance problem; however, for this study, it is assumed that it plays a minimum role. Education in the current study $\left(R_{\mathrm{ed}}\right)$ includes basic knowledge on self-hygiene, the importance of using toilets, drinking boiled water, educating humans not to contaminate water, use of oral salts to help already infected individuals, avoiding direct contact with infected individuals, etc. It can be further noticed from Figure 2(a) that education campaign is more important than sanitation because individual awareness about the disease limits the spread of the epidemic better. The last scenario is sanitation $\left(R_{S}\right)$ which principally entails water sanitation. Treating water with chlorine plays a vital role in combating Shigellosis. This is because the addition of chlorine in water kills Shigella bacteria. Even though treatment offers the best results among the single strategies, its implementation is not practical for most communities, especially in the developing world where medical facilities are still problematic due to financial constraints. Furthermore, most Shigellosis antibiotics are resistant to treat this disease. Such constraints hinder Shigellosis eliminations in most communities. Therefore, other options of control strategies such as sanitation and education could be successfully applied in the absence of treatment and still bring forth promising results. That is why many governments today opt to offer clean water to its populace because it is not only cheaper but also healthier than treatment. In so doing they tend to limit the eruption of many waterborne diseases including, Shigellosis. 


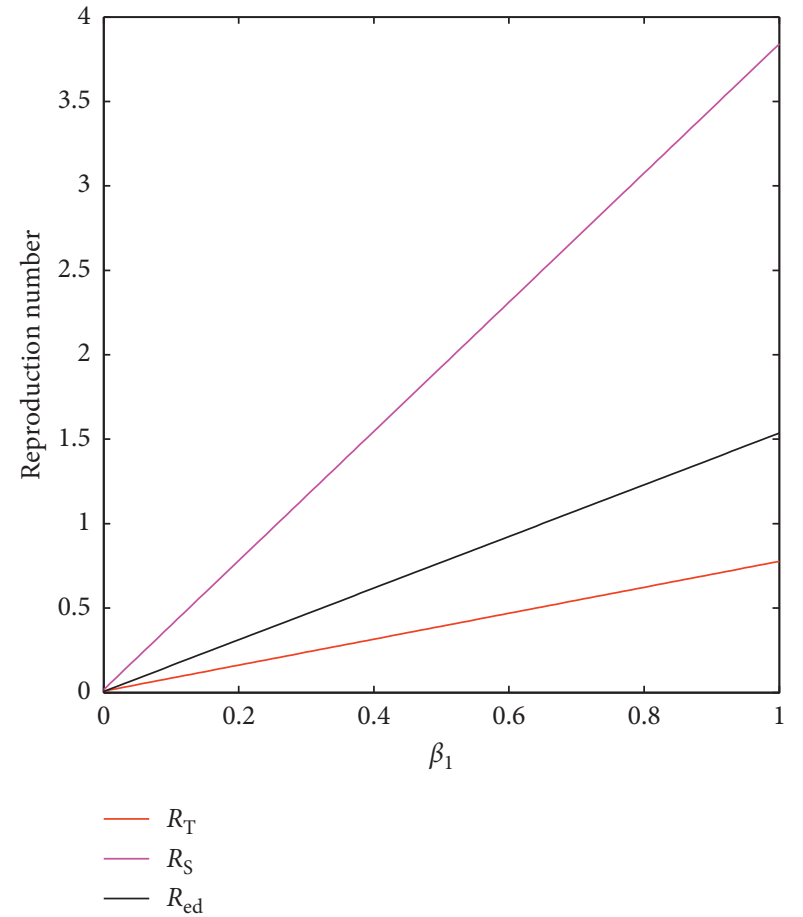

(a)

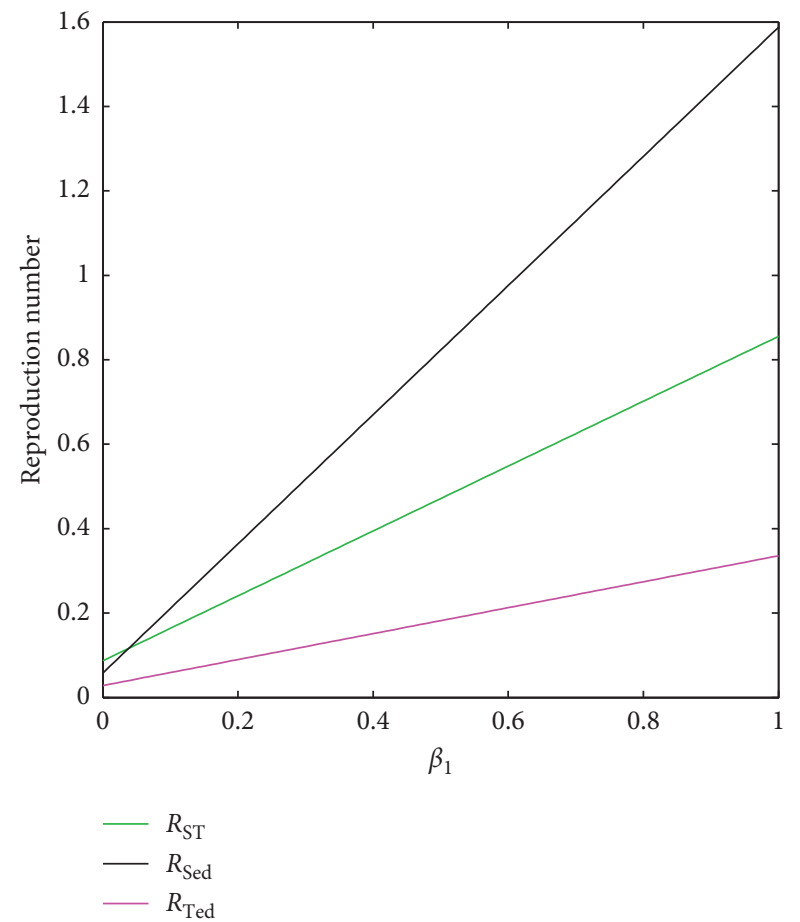

(b)

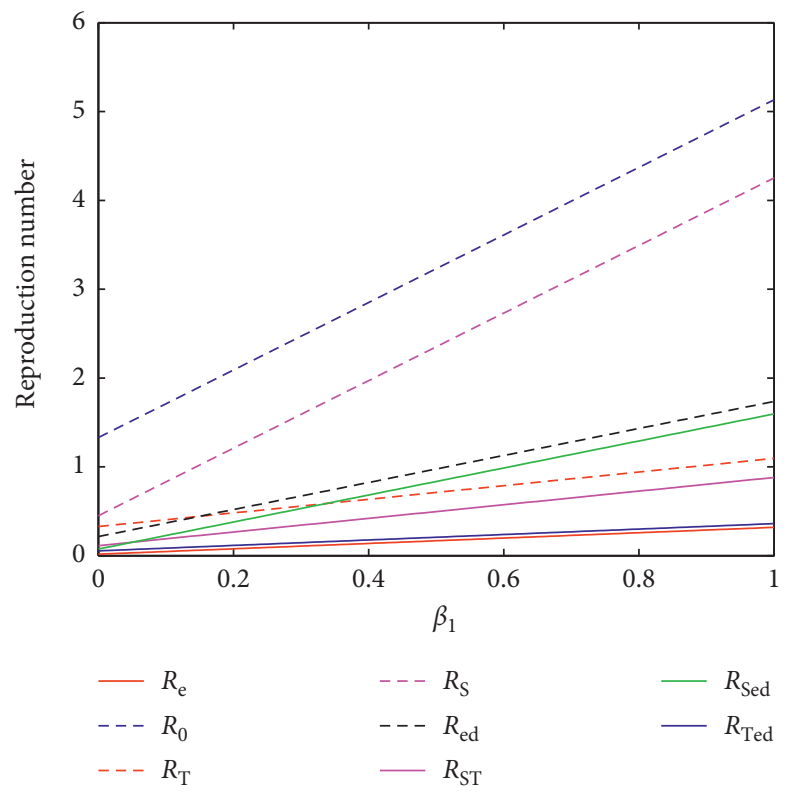

(c)

Figure 2: The comparison of single strategies (a), double strategies (b), and three control strategies (c). Basic reproduction number $\left(R_{0}\right)$ is the largest followed by single induced reproduction numbers and then the dual controls and the least being reproduction number capturing all three controls $\left(R_{e}\right)$. Note that reproduction number with education and treatment efforts $\left(R_{\mathrm{Ted}}\right)$ has coincided with $R_{e}$.

In addition to that, Figure 2(b) shows that a combination of treatment and education campaign $\left(R_{\text {Ted }}\right)$ yields the best results among the dual strategies followed by the combination of sanitation and treatment $\left(R_{\mathrm{ST}}\right)$ and the last combination being sanitation and education $\left(R_{\text {Sed }}\right)$.

Also, it can be seen from Figure 2(c) that as the number of control strategies increase from none to three control strategies, there is a significant decrease in disease eruption. Our results show that when all three interventions are implemented simultaneously, then disease control is possible. Further, it can be noted that the inclusion of treatment either singly or a combination is more beneficial as compared to when it is excluded (see $R_{T}$ and $R_{\text {Sed }}$ ) in Figure 2(c). 


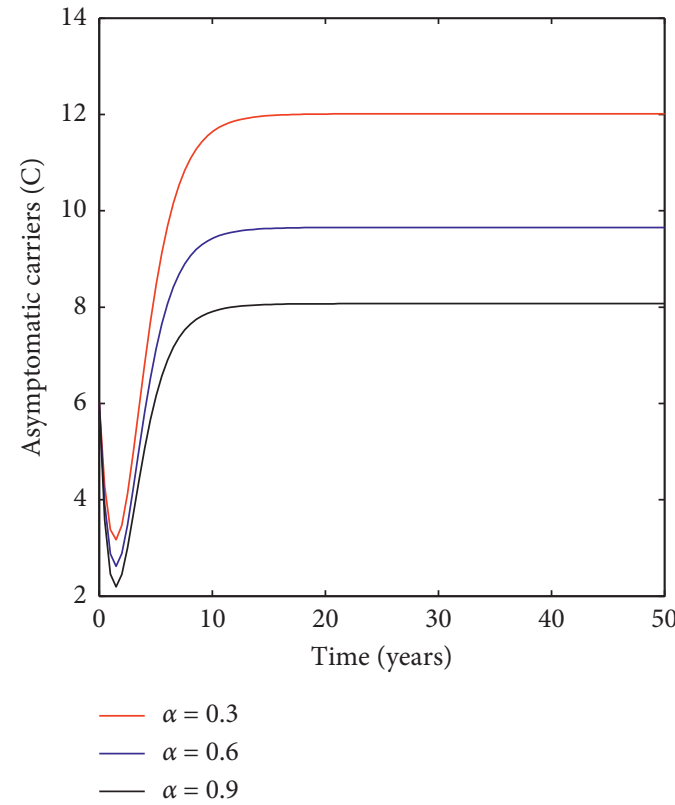

(a)

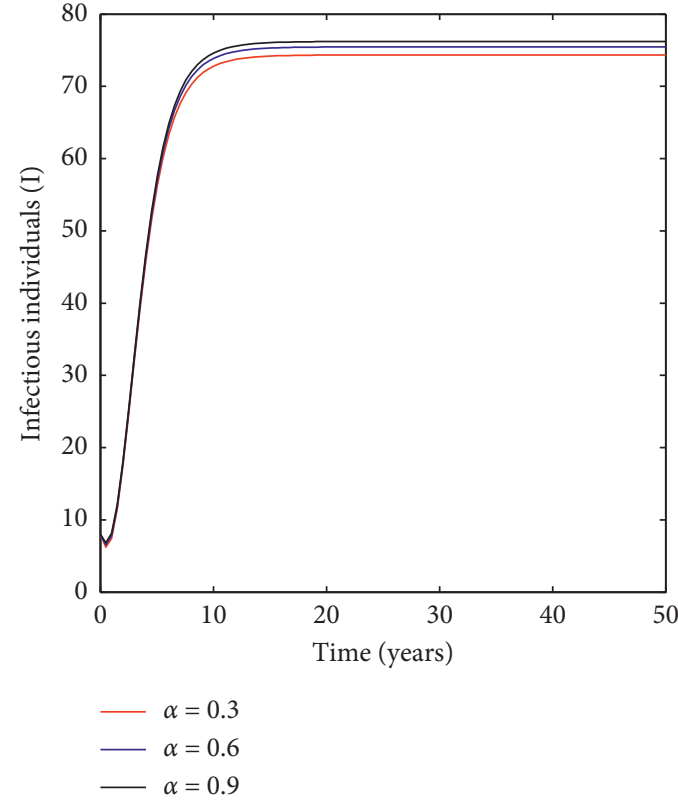

(b)

Figure 3: (a, b) Simulation results showing the impact of testing and diagnosis of asymptomatic carriers and symptomatic infectious individuals.

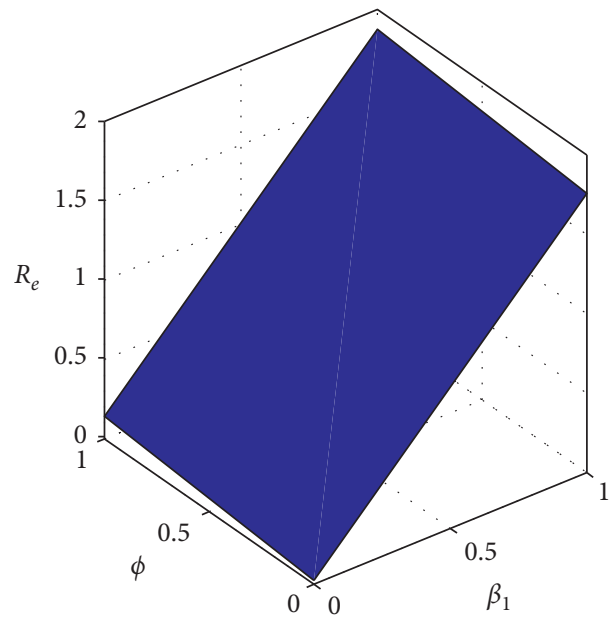

(a)

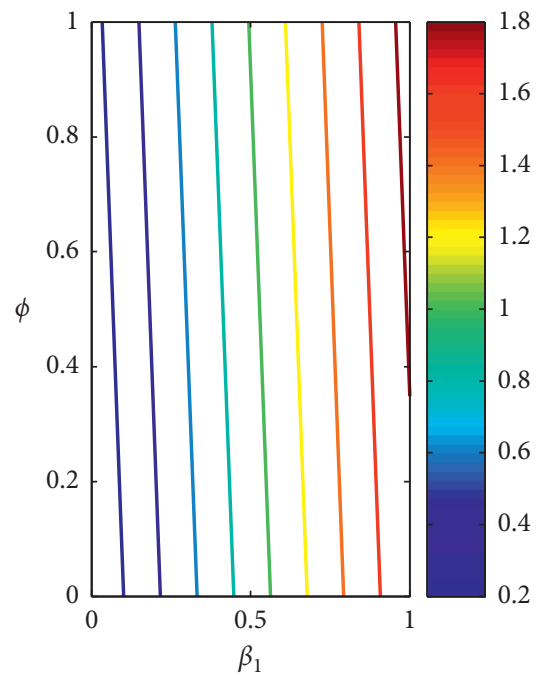

(b)

Figure 4: (a, b) As transmission rates $\phi$ and $\beta_{1}$ increase, there is an increase in disease epidemic. More people are infected with Shigellosis as a result of exposure to the environment or infectious people.

Further simulations of model (2) with controls measures are depicted in Figure 5 which shows that an increase in each of the control strategies: education $(\rho)$, treatment $(\gamma)$, screening $(\alpha)$, and sanitation $(\sigma)$,has an impact in reducing the number of Shigellosis cases from individuals or the surroundings. Figure 5(a) shows that when education coverage increases from $30 \%$ to $90 \%$, more individuals remain susceptible since they tend to avoid getting new infections or infecting others. Also, from Figure 5(b), one can note that varying treatment rate from $30 \%$ to $90 \%$ decreases the size of infectious individuals; this suggests the significance of treatment in breaking further transmission to the safe populace. In the same manner, Figure 5(c) shows that as sanitation rate increases from $30 \%$ to $90 \%$, there is a significant decline of Shigella bacteria as they are killed from the surroundings. This suggests the significance of undertaking sanitation if we are to bring this epidemic to an end. On the other hand, identification of carriers for Shigella 


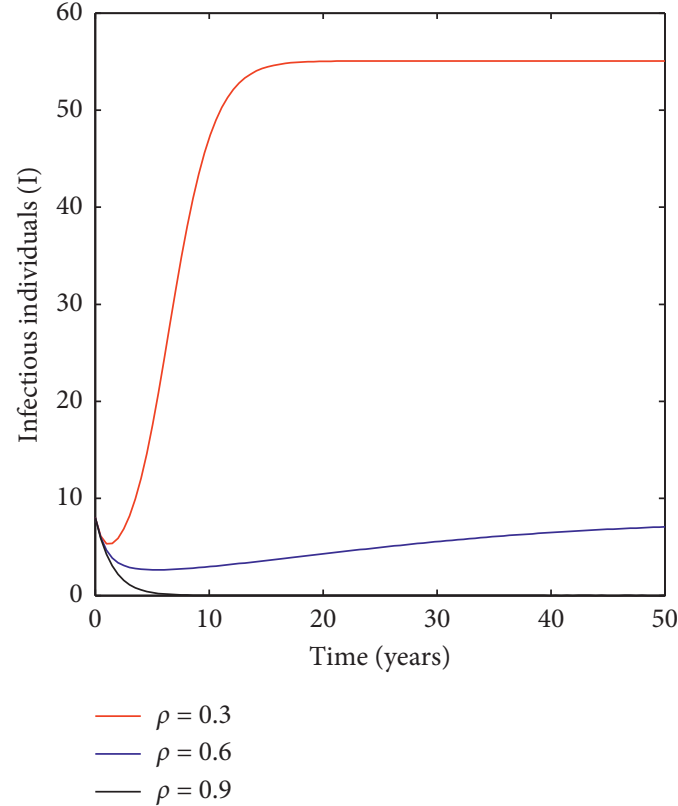

(a)

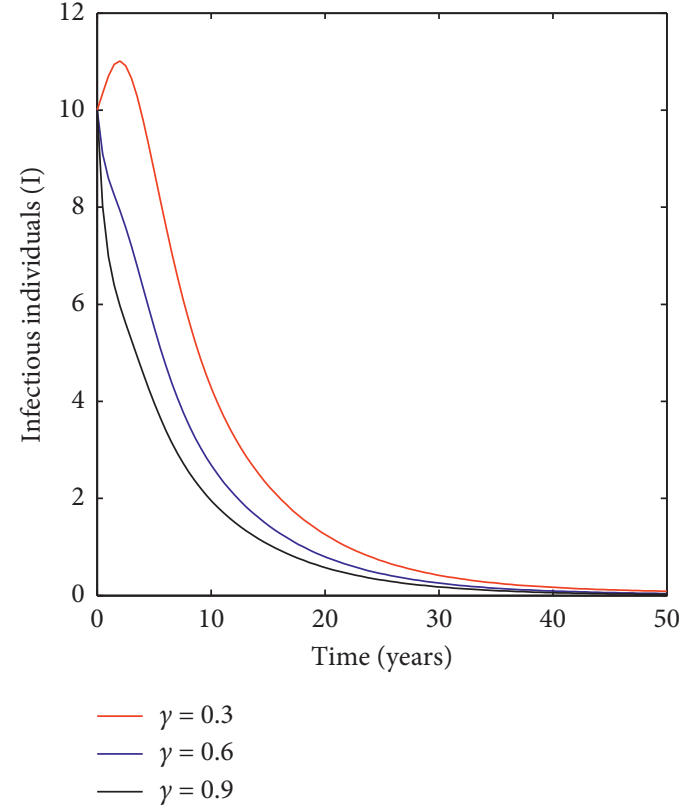

(b)

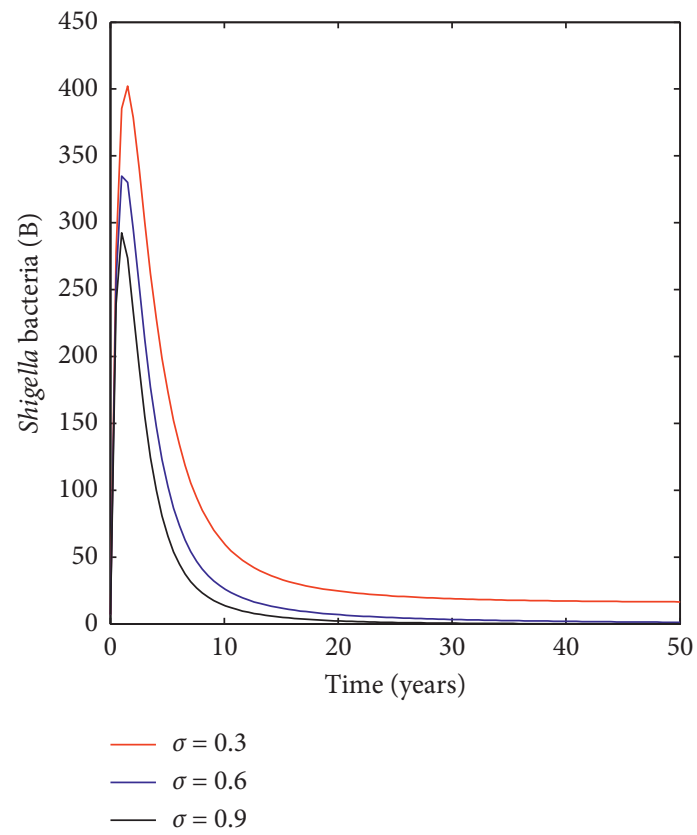

(c)

FIGURE 5: Effects of controls on the susceptible (a), symptomatic infectious patients, (b) and Shigella bacteria population (c).

bacteria is beneficial since the identified population will be treated to safeguard their life and life of others.

\section{Sensitivity Analysis}

Sensitivity analysis describes how the uncertainty on the model inputs influences the model output. There are two main classes of sensitivity analysis method based on local or global definitions. Both approaches will be described in this section.
6.1. Local Sensitivity Analysis. Local sensitivity analysis assesses the effects of individual parameters at particular points in parameter space without taking into account the combined variability resulting from considering all input parameters simultaneously.

Local sensitivity for model (2) will be determined via the reproduction number $R_{e}$ given by equation (28). Since $R_{e}$ depends only on 21 parameters, we derive an analytical expression for its sensitivity to each parameter using the normalized forward sensitivity index as done in [28] as follows: 


$$
\begin{aligned}
\Gamma_{\Lambda}^{R_{e}} & =\frac{\partial R_{e}}{\partial \Lambda} \times \frac{\Lambda}{R_{e}}=+1, \\
\Gamma_{\mu_{h}}^{R_{e}} & =\frac{\partial R_{e}}{\partial \mu_{h}} \times \frac{\mu_{h}}{R_{e}}=-1.0020 .
\end{aligned}
$$

The rest of sensitivity indices for all parameters used in equation (28) can be computed in a similar approach. Table 3 shows the sensitivity indices of $R_{e}$ with respect to 21 parameters.

From Table 3, we can obtain $\Gamma_{\mu_{h}}^{R_{e}}=-1.002$; this means that an increase in $\mu_{h}$ will cause a decrease in $R_{e}$. Similarly, a decrease in $\mu_{h}$ will cause an increase in $R_{e}$, as they are inversely proportional. We can also note that $q, l, \alpha, d_{1}, \eta_{1}, \eta_{2}, K, \mu_{b}, \gamma, \rho$, and $\sigma$ are all negative; hence, these parameters are inversely proportional to $R_{e}$. This means that an increase (decrease) in any of these parameters will cause a decrease (increase) in $R_{e}$.

On the other hand, one can see that $\Gamma_{\Lambda}^{R_{e}}=+1$; this means that an increase in $\Lambda$ will cause an increase of exactly the same proportion in $R_{e}$. Correspondingly, a decrease in $\Lambda$ will cause a decrease in $R_{e}$, as they are directly proportional. We can also note that $\beta_{1}, \beta_{2}, \phi, \delta, \epsilon_{1}, \epsilon_{2}$, or $r>0$; hence, these parameters are directly proportional to $R_{e}$. This means that an increase (decrease) in any of these parameters will cause an increase (decrease) in $R_{e}$. We can arrange these parameters in the order of their magnitude from the largest to the smallest as follows: $\mu_{h}, \Lambda, q, \beta_{2}, \beta_{1}, \rho, \alpha, \eta_{1}, \phi, K, \gamma, \mu_{b}, \eta_{2}$, $\sigma, \epsilon_{1}, r, \epsilon_{2}, l, d_{1}$ and the least sensitive parameter is $\delta$. The effective reproduction number $R_{e}$ decreases with an increase in the parameters catering for control measures $(\sigma, \rho, \gamma, \alpha)$. This suggests that an optimal control measure could be the suitable combination of sanitation, education campaigns to a broad audience, the screening of asymptomatic carriers, and the treatment of symptomatic infectious individuals.

More precisely, to minimize Shigellosis transmission in a population, this study recommends that the general public should avoid visiting endemic areas $(\Lambda)$, reducing unnecessary contact with infectious and carriers to reduce the transmission rates $\left(\beta_{1}, \beta_{2}\right)$, increase Shigellosis specific education $(\rho)$ on its transmission and control, and avoid contact with contaminated food or water; this will reduce the transmission rate $(\phi)$ as such contacts increase the chance of contracting this epidemic. Likewise, screening of the carriers $(\alpha)$ would help to contain the spread of the disease as screened individuals will receive medical attention immediately after being identified, and increased treatment rates $(\gamma)$ of already infected individuals will break the chain of transmission. Keeping our bodies healthier has an additional advantage of increasing natural immune system which would boost natural recovery $\left(\eta_{1}, \eta_{2}\right)$, and people need to keep their bodies healthier by opting to a healthy lifestyle (eating a balanced diet and regular exercises). Increased environmental sanitation $(\sigma)$ has benefits of killing bacteria; efforts such as water purification and safe disposal of wastes would deteriorate bacterial in the environment. Growth rate $(r)$ tends to increase bacterial concentration in the
TABle 3: Parameters and their sensitivity indices.

\begin{tabular}{lc}
\hline Parameter & Sensitivity index \\
\hline$\Lambda$ & +1.0000 \\
$\beta_{1}$ & +0.3344 \\
$\beta_{2}$ & +0.4101 \\
$\phi$ & +0.2555 \\
$\delta$ & +0.0013 \\
$\mu_{h}$ & -1.0021 \\
$q$ & -0.6524 \\
$l$ & -0.0158 \\
$\alpha$ & -0.2776 \\
$d_{1}$ & -0.0105 \\
$\eta_{1}$ & -0.2625 \\
$\eta_{2}$ & -0.2009 \\
$K$ & -0.2555 \\
$\epsilon_{1}$ & +0.1489 \\
$\epsilon_{2}$ & +0.1065 \\
$r$ & +0.1433 \\
$\mu_{b}$ & -0.2077 \\
$\gamma$ & -0.2100 \\
$\rho$ & -0.3139 \\
$\sigma$ & -0.1911 \\
\hline
\end{tabular}

TABle 4: Parameter value ranges of model (1) used as input for the LHS method.

\begin{tabular}{lc}
\hline Parameter & Range \\
\hline$\Lambda$ & {$[500,1500]$} \\
$\beta_{1}$ & {$[0.2,0.6]$} \\
$\beta_{2}$ & {$[0.3,0.8]$} \\
$\phi$ & {$[0.2465,0.6465]$} \\
$\delta$ & {$[0.15,0.35]$} \\
$\mu_{h}$ & {$[0.000257,0.000457]$} \\
$q$ & {$[0.3,0.8]$} \\
$l$ & {$[0.2,0.5]$} \\
$\alpha$ & {$[0.26,0.6]$} \\
$d_{1}$ & {$[0.01,0.04]$} \\
$d_{2}$ & {$[0.01,0.05]$} \\
$\eta_{1}$ & {$[0.2,0.8]$} \\
$\eta_{2}$ & {$[0.2,0.6]$} \\
$K$ & {$[100,300]$} \\
$\epsilon_{1}$ & {$[40,80]$} \\
$\epsilon_{2}$ & {$[30,80]$} \\
$r$ & {$[0.25,0.5]$} \\
$\mu_{b}$ & {$[0.252086,0.852086]$} \\
$\gamma$ & {$[0.2,0.6]$} \\
$\rho$ & {$[0.1,0.3]$} \\
$\sigma$ & {$[0.1,0.8]$} \\
\hline
\end{tabular}

environment, so efforts to reduce this rate should be made. Defecation in water sources done by infectious individuals or carriers, i.e., $\epsilon_{1}, \epsilon_{2}$, should be discouraged as this tends to amplify Shigella bacteria in the environment.

6.2. Uncertainty and Global Sensitivity Analysis. Global sensitivity analysis is the process of apportioning the uncertainty in outputs to the uncertainty in each input factor over their entire range of interest. A sensitivity analysis is 


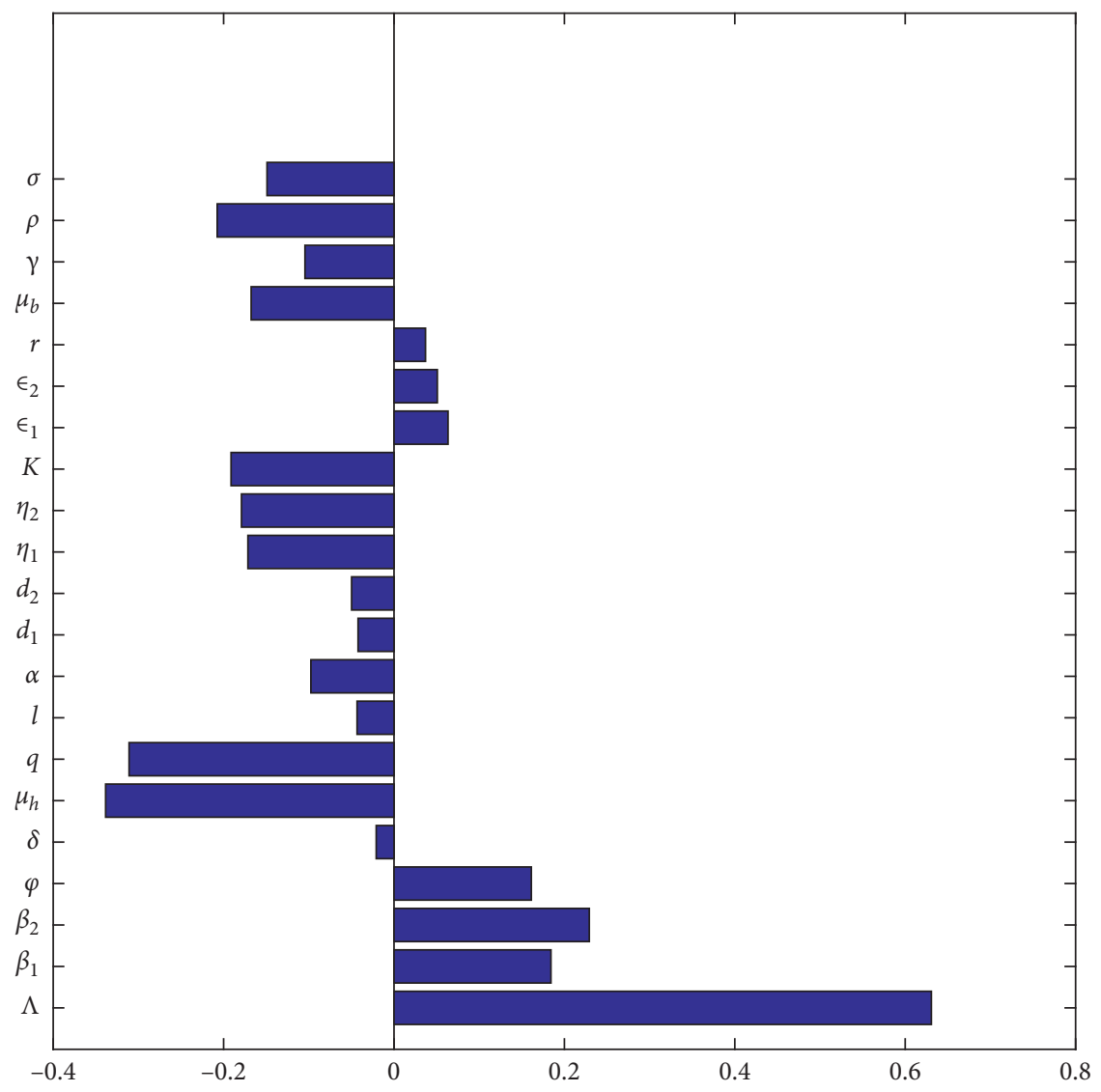

FIgURE 6: Tornado plots of PRCC of parameters that influence model (2) generated using parameter values in Table 4. Parameters with PRCC $>0$ and PRCC $<0$ increase and decrease values of $R_{e}$, respectively.

considered to be global when all the input factors are varied simultaneously and the sensitivity is evaluated over the entire range of each input factor [29]. Here, we perform a global sensitivity analysis to examine the model responses to parameter variation within a wider range in the parameter space. The mean values of parameters are listed in Table 3, and the range values of these parameters are given in Table 4.

It is important to notice that variations of these parameters in our deterministic model lead to uncertainty to model predictions since the effective reproductive number varies with parameters. We adopted the approach in [30]; partial rank correlation coefficients (PRCCs) between the effective reproduction number $R_{e}$ and each parameter for model (2) are derived and are shown in Figure 6. Due to the absence of data on the distribution function, a uniform distribution is chosen for all parameters. The sets of input parameter values sampled using the Latin hypercube sampling (LHS) method were used to run 1000 simulations. We compute the partial rank correlation coefficients between $R_{e}$ and each parameter of model (2). The results of the PRCC are shown in Table 5. It must be noted that the parameters with large PRCC values are most influential in model (1). Table 5 shows that the parameter $\Lambda$ has the highest influence on the reproduction number $R_{e}$, followed in decreasing order by the parameters $\mu_{h}, q, K, \eta_{1}, \beta_{2}, \sigma, \beta_{1}, \mu_{b}, \phi, \eta_{2}, \rho, \epsilon_{2}$, and $\gamma$.
TABle 5: Parameters and their sensitivity indices.

\begin{tabular}{lc}
\hline Parameter & PRCCs \\
\hline$\Lambda$ & +0.597 \\
$\beta_{1}$ & +0.184 \\
$\beta_{2}$ & +0.195 \\
$\phi$ & +0.148 \\
$\delta$ & +0.00549 \\
$\mu_{h}$ & -0.371 \\
$q$ & -0.267 \\
$l$ & -0.021 \\
$\alpha$ & -0.0784 \\
$d_{1}$ & -0.0163 \\
$d_{2}$ & -0.065 \\
$\eta_{1}$ & -0.198 \\
$\eta_{2}$ & -0.144 \\
$K$ & -0.204 \\
$\epsilon_{1}$ & +0.0305 \\
$\epsilon_{2}$ & +0.104 \\
$r$ & +0.0768 \\
$\mu_{b}$ & -0.174 \\
$\gamma$ & -0.102 \\
$\rho$ & -0.125 \\
$\sigma$ & -0.194 \\
\hline
\end{tabular}

The other parameters are $\alpha, r, d_{2}, \epsilon_{1}, l, d_{1}$, and $\delta$. These parameters have almost no effect on $R_{e}$. It can be noted that parameters such as $\beta_{2}, \beta_{1}, \sigma, \rho$, and $\gamma$ allow us to considerably 
reduce the reproduction number. Hence, the sensitivity analysis consistently reinforces our suggestion that the most effective manner to reduce infection is to increase education campaigns, sanitation, and treatment strategies. It can be noted that the order of the most important parameters for $R_{e}$ from the local sensitivity analysis does not match those from the global sensitivity analysis; this shows that the local results are not robust enough.

\section{Conclusion}

In the current work, we have developed a deterministic mathematical model for transmission dynamics of Shigellosis that captures both direct and indirect transmission. The model has incorporated multiple interventions in the presence of carriers. From the model, we have derived the effective reproduction number using the next-generation matrix method, and we have used it to establish the stability of the equilibrium points. We derived both the disease-free equilibrium and the endemic equilibrium. We proved that the DFE is locally asymptotically stable (l.a.s) when $R_{e}<1$, whereas the EE was proved to be globally asymptotically stable when $R_{e}>1$. The model developed included intervention strategies such as sanitation, treatment, and education campaigns, and our results have shown that the integration of these control strategies into the model either singly or combined is beneficial to clear away Shigellosis epidemic.

More than that, we have performed a sensitivity analysis using the effective reproduction number to determine the contribution of each parameter to this reproduction number. Most sensitive parameters are the human mortality rate $\left(\mu_{h}\right)$, recruitment rate $(\Lambda)$, a fraction of exposed individuals who progress to infectious class $(q)$, the transmission rate for carriers $\left(\beta_{2}\right)$, the transmission rate for infectious individuals $\left(\beta_{1}\right)$, education efficacy $(\rho)$, and screening and testing $(\alpha)$. These parameters need great attention if one needs to eradicate Shigellosis from the community. Other parameters are natural recovery for infectious individuals $\left(\eta_{1}\right)$, human-to-environmental transmission rate $(\phi)$, half-saturation constant of Shigella bacteria that can cause a $50 \%$ chance of infection $(K)$, treatment rate of infectious individuals $(\gamma)$, Shigella death rate $\left(\mu_{b}\right)$, natural recovery for infectious carriers $\left(\eta_{2}\right)$, sanitation rate $(\sigma)$, contribution by infectious individuals $\left(\epsilon_{1}\right)$, Shigella growth rate $(r)$, contribution by carriers $\left(\epsilon_{2}\right)$, a fraction of carriers who recover naturally $(l)$, infectioninduced death rate $\left(d_{1}\right)$, and rate of incubation $(\delta)$.

One of our impressive results is that direct transmission between human-to-human $\left(\beta_{1}, \beta_{2}\right)$ contributes more to Shigella infection as compared with the indirect transmission $(\phi)$; the same result was reported in [31,32]. Apart from that, sensitivity analysis shows that carriers are more infectious than symptomatic infectious individuals (see sensitivity indices for $\beta_{1}$ and $\beta_{2}$ ). It might be because carriers continue infecting others unknowingly as they do not know their status, and so susceptibles cannot take any precautionary measures against the carriers. By this way, transmission from carriers to the susceptibles becomes apparent.
On the other hand, simulations show that treatment strategy has a more significant impact of reducing infections as compared with other strategies such as education campaigns and sanitation. However, treatment has economic and drug resistance implications.

\section{Data Availability}

The data used to support the findings of this study are available from the corresponding author upon request.

\section{Conflicts of Interest}

The authors declare that they have no conflicts of interest.

\section{Acknowledgments}

Stephen Edward appreciates the financial support from the University of Dodoma (UDOM) during the study.

\section{References}

[1] A. A. Lindberg, P. D. Cam, N. Chan et al., "Shigellosis in vietnam: seroepidemiologic studies with use of lipopolysaccharide antigens in enzyme immunoassays," Clinical Infectious Diseases, vol. 13, no. Supplement_4, pp. S231-S37, 1991.

[2] G. N. Schroeder and H. Hilbi, "Molecular pathogenesis of Shigella spp.: controlling host cell signaling, invasion, and death by type III secretion," Clinical Microbiology Reviews, vol. 21, no. 1, pp. 134-156, 2008.

[3] K. L. Kotloff, J. P. Winickoff, B. Ivanoff et al., "Global burden of Shigella infections: implications for vaccine development and implementation of control strategies," Bulletin of the World Health Organization, vol. 77, no. 8, pp. 651-66, 1999.

[4] F. Scheutz, N. A. Strockbine, G. M. Garrity, D. J. Brenner, N. R. Krieg, and J. T. Staley, Bergey's Manual of Systematic Bacteriology, pp. 607-613, Springer Publishing, New York, NY, USA, 2005.

[5] C.-S. Chiou, W.-B. Hsu, H.-L. Wei, and J.-H. Chen, "Molecular epidemiology of a Shigella flexneri outbreak in a mountainous township in Taiwan, Republic of China," Journal of Clinical Microbiology, vol. 39, no. 3, pp. 1048-1056, 2001.

[6] D. L. Martin, T. L. Gustafson, J. W. Pelosi, L. Suarez, and G. V. Pierce, "Contaminated produce-a common source for two outbreaks of Shigella gastroenteritis," American Journal of Epidemiology, vol. 124, no. 2, pp. 299-305, 1986.

[7] G. Samonis, L. Elting, E. Skoulika, S. Maraki, and Y. Tselentis, "An outbreak of diarrhoeal disease attributed to Shigella sonnei," Epidemiology and Infection, vol. 112, no. 2, pp. 235-245, 1994.

[8] W. Swaddiwudhipong, S. Karintraratana, and S. Kavinum, “A common-source outbreak of shigellosis involving a piped public water supply in Northern Thai communities," The Journal of Tropical Medicine and Hygiene, vol. 98, no. 3, pp. 145-150, 1995.

[9] P. S. Mead, L. Slutsker, V. Dietz et al., "Food-related illness and death in the United States," Emerging Infectious Diseases, vol. 5, no. 5, pp. 607-625, 1999.

[10] F. He, K. Han, L. Liu et al., "Shigellosis outbreak associated with contaminated well water in a rural elementary school: Sichuan Province, China, June 7-16, 2009," PLoS One, vol. 7, no. 10, Article ID e47239, 2012. 
[11] A. K Bhunia, "Shigella species," in Foodborne Microbial Pathogens: Mechanisms and Pathogenesis, J. L. S. Golden, A. David, W. H Richard et al., Eds., pp. 253-264, SpringerVerlag, New York, NY, USA, 2008.

[12] World Health Organization, Guidelines For the Control of Shigellosis, Including Epidemics Due to Shigella dysenteriae Type 1, World Health Organization, Geneva, Switzerland, 2005.

[13] W. Zhang, Y. Luo, J. Li et al., "Wide dissemination of multidrug-resistant Shigella isolates in China," Journal of Antimicrobial Chemotherapy, vol. 66, no. 11, pp. 2527-2535, 2011.

[14] M. M. Levine, K. L. Kotloff, E. M. Barry, M. F. Pasetti, and M. B. Sztein, "Clinical trials of Shigella vaccines: two steps forward and one step back on a long, hard road," Nature Reviews Microbiology, vol. 5, no. 7, pp. 540-553, 2007.

[15] A. Phalipon, C. Costachel, C. Grandjean et al., "Characterization of functional oligosaccharide mimics of the Shigella flexneri serotype 2a O-antigen: implications for the development of a chemically defined glycoconjugate vaccine," The Journal of Immunology, vol. 176, no. 3, pp. 1686-1694, 2006.

[16] J. H. Tien and D. J. D. Earn, "Multiple transmission pathways and disease dynamics in a waterborne pathogen model," Bulletin of Mathematical Biology, vol. 72, no. 6, pp. 1506-1533, 2010.

[17] O. Chaturvedi, T. Masupe, and S. Masupe, "A continuous mathematical model for Shigella outbreaks," American Journal of Biomedical Engineering, vol. 4, no. 1, pp. 10-16, 2014.

[18] T. Chen, R. K. K. Leung, Z. Zhou, R. Liu, X. Zhang, and L. Zhang, "Investigation of key interventions for shigellosis outbreak control in China," PLoS One, vol. 9, no. 4, Article ID e95006, 2014.

[19] H. W. Berhe, O. D. Makinde, and D. M. Theuri, "Parameter estimation and sensitivity analysis of dysentery diarrhea epidemic model," Journal of Applied Mathematics, vol. 2019, Article ID 8465747, 13 pages, 2019.

[20] C. T. Codeco, "Endemic and epidemic dynamics of cholera: the role of the aquatic reservoir," BMC Infectious Diseases, vol. 1, no. 1, p. 1, 2001.

[21] A. Mwasa and J. M. Tchuenche, "Mathematical analysis of a cholera model with public health interventions," Biosystems, vol. 105, no. 3, pp. 190-200, 2011.

[22] O. C. Collins and K. J. Duffy, "Analysis and optimal control intervention Strategies of a waterborne disease model: a realistic case study," Journal of Applied Mathematics, vol. 2018, Article ID 2528513, 14 pages, 2018.

[23] O. Diekmann, J. A. P. Heesterbeek, and M. G. Roberts, "The construction of next-generation matrices for compartmental epidemic models," Journal of the Royal Society Interface, vol. 7, no. 47, pp. 873-885, 2009.

[24] N. Shaban, "Modelling the Effects of public health education in the spread of hepatitis B disease," Applied Mathematical Sciences, vol. 9, no. 80, pp. 3967-3981, 2015.

[25] A. Korobeinikov and G. C. Wake, "Lyapunov functions and global stability for SIR, SIRS, and SIS epidemiological models," Applied Mathematics Letters, vol. 15, no. 8, pp. 955-960, 2002.

[26] A. Korobeinikov, "Global properties of basic virus dynamics models," Bulletin of Mathematical Biology, vol. 66, no. 4, pp. 879-883, 2004.

[27] A. Korobeinikov, "Global properties of infectious disease models with nonlinear incidence," Bulletin of Mathematical Biology, vol. 69, no. 6, pp. 1871-1886, 2007.
[28] N. Chitnis, J. M. Hyman, and J. M. Cushing, "Determining important parameters in the spread of malaria through the sensitivity analysis of a mathematical model," Bulletin of Mathematical Biology, vol. 70, no. 5, pp. 1272-1296, 2008.

[29] X. Zhou, H. Lin, and H. Lin, "Global sensitivity analysis," in Encyclopedia of GIS, S. Shekhar and H. Xiong, Eds., Springer, Boston, MA, USA, 2008.

[30] S. Marino, I. B. Hogue, C. J. Ray, and D. E. Kirschner, "A methodology for performing global uncertainty and sensitivity analysis in systems biology," Journal of Theoretical Biology, vol. 254, no. 1, pp. 178-196, 2008.

[31] J. C. Mohle-Boetani, M. Stapleton, R. Finger et al., "Communitywide shigellosis: control of an outbreak and risk factors in child day-care centers," American Journal of Public Health, vol. 85, no. 6, pp. 812-816, 1995.

[32] M. Malengreau, M. Kaba, M. Gillieaux, M. De Feyter, and M. Duibone, "Outbreak of Shigella dysentery in eastern Zaire, 1980-1982," Annales de la Societe Belge de Medecine Tropicale, vol. 63, no. 1, pp. 59-67, 1983. 